\title{
Information transfer fidelity in spin networks and ring-based quantum routers
}

\author{
E. Jonckheere ${ }^{1}$ - F. C. Langbein ${ }^{2}$ - S. G. Schirmer ${ }^{3}$
}

Received: 9 May 2015 / Accepted: 23 September 2015 / Published online: 8 October 2015

(C) The Author(s) 2015. This article is published with open access at Springerlink.com

\begin{abstract}
Spin networks are endowed with an information transfer fidelity (ITF), which defines an absolute upper bound on the probability of transmission of an excitation from one spin to another. The ITF is easily computable, but the bound can be reached asymptotically in time only under certain conditions. General conditions for attainability of the bound are established, and the process of achieving the maximum transfer probability is given a dynamical model, the translation on the torus. The time to reach the maximum probability is estimated using the simultaneous Diophantine approximation, implemented using a variant of the Lenstra-Lenstra-Lovász (LLL) algorithm. For a ring with uniform couplings, the network can be made into a metric space by defining a distance (satisfying the triangle inequality) that quantifies the lack of transmission fidelity between two nodes. It is shown that transfer fidelities and transfer times can be improved significantly by means of simple controls taking the form of nondynamic, spatially localized bias fields, opening up the possibility for intelligent design of spin networks and dynamic routing of information encoded in them, while being more flexible than engineering fixed couplings to favor some transfers, and less demanding than control schemes requiring fast dynamic controls.
\end{abstract}

$凶 \quad$ E. Jonckheere

jonckhee@usc.edu

F. C. Langbein

LangbeinFC@cardiff.ac.uk

S. G. Schirmer

sgs29@swan.ac.uk

1 Center for Quantum Information Science and Technology, University of Southern California, Los Angeles, CA 90089, USA

2 School of Computer Science and Informatics, Cardiff University, Cardiff CF24 3AA, UK

3 College of Science (Physics), Swansea University, Singleton Park, Swansea SA2 8PP, UK 
Keywords Spin networks · Quantum ring routers · Information transfer fidelity · Simultaneous Diophantine approximation · Lenstra-Lenstra-Lovász (LLL) lattice basis reduction · Information geometry

\section{Introduction}

Efficient and controllable transport of information is crucial for information processing, both classical and quantum. While bosonic channels [1] are the most attractive option for long-distance communication, efficient on-chip interconnectivity in a quantum processor based on atomic, ionic or quantum dot-based qubits, or quantum spintronic devices [2], will require direct information transport through networks of coupled solid-state qubits. Such networks can be modeled via interacting spins and are therefore generally referred to as spin networks. Initiated by Bose's [3] seminal work, spin networks have received considerable attention in recent years (see review articles [4,5] and references therein). Most of the work has focused on information transmission through linear chains as prototype quantum wires, starting with unmodulated chains [3] and later perfect state transfer in chains with fixed, engineered couplings [6,7], and finally controlled state transfer in spin chains, e.g., via adiabatic passage [8], ac modulation to achieve renormalization of the couplings between adjacent qubits [9], single-node bang-bang controls [10] or global dynamic controls [11]. Perfect state transfer in more general networks has also been considered, and some interesting results for complete graphs were obtained in [12].

Nonetheless, the information-theoretic properties of spin networks are not fully understood. Information encoded in excitations of a network of coupled spins propagates, even under ideal conditions when quantum coherence is maintained, in a nonclassical way determined by the Schrödinger equation. Under the best possible circumstances, this propagation of excitations determines the information transfer fidelity (ITF) between various nodes of the network. ${ }^{1}$ Perfect state transfer between two nodes can only be achieved when the information transfer fidelity between the respective nodes is unity. However, this condition is not sufficient. For example, while it is satisfied for the end nodes of a chain with uniform couplings [13], such chains are usually not considered to admit perfect state transfer except for chains of length two or three.

This raises the question of the attainability of the upper bound given by the information transfer fidelity. Attainability in general also depends on time constraints, i.e., attainable in what time, and the margins of errors we are willing to accept. In practice, some margin of error is unavoidable, and the real question of interest is therefore not whether we can achieve, e.g., perfect, i.e., unit fidelity, state transfer in time $t_{f}$, but rather whether we can achieve state transfer with a fidelity $1-\epsilon$, where $\epsilon$ is an acceptable margin of error, in a reasonable amount of time. We may be willing to accept a slightly increased margin of error for a significant reduction in the transfer time. In this work, we are interested in such fundamental questions for spin networks

\footnotetext{
1 Previously [13], this concept was named information transfer capacity, but we refrain here from using this terminology to avoid confusion with the Shannon channel capacity [1].
} 
subject to coherent dynamics in general, and specifically simple configurations such as a circular arrangement of spins (or spin ring for short), which could serve as basic building blocks for more complex architectures.

After introducing some basic definitions and basic results in Sect. 2, the concept of asymptotic ITF, i.e., maximum information transfer fidelity attainable absent constraints on the transfer times, between nodes in a network of spins is introduced in Sect. 3. Conditions for attainability of the bounds are derived using dynamic flows on tori and the simultaneous Diophantine approximation, computationally implemented using the Lenstra-Lenstra-Lovász (LLL) algorithm. Under certain conditions, the information transfer infidelity induces a metric that captures how close two nodes in a spin network are from an information-theoretic point of view. This information transfer geometry is investigated in Sect. 4. Finally, in Sect. 5, we investigate how the information transfer geometry of a network can be changed by means of simple controls in the form of fixed biases applied to individual nodes and how this principle could be employed for dynamic routing in a spin network with ring topology without the requirement of fast-switching controls.

\section{Basic definitions and results}

We consider networks of $N$ spins arranged in some regular pattern with either $\mathrm{XX}$ or Heisenberg interaction [14] specified by the Hamiltonian

$$
H=\sum_{i, j=1}^{N} J_{i j}\left(\sigma_{i}^{x} \sigma_{j}^{x}+\sigma_{i}^{y} \sigma_{j}^{y}+\eta \sigma_{i}^{z} \sigma_{j}^{z}\right) .
$$

We specifically focus on networks with XX coupling $(\eta=0)$ and Heisenberg coupling $(\eta=1)$, although most of the concepts and analysis in the following are not limited to these types of coupling. $J_{i j}$ is the strength of the coupling between spin $i$ and spin $j$. The factor $\sigma_{i}^{x, y, z}$ is the Pauli matrix along the $x, y$, or $z$ direction of spin $i$, i.e.,

$$
\sigma_{i}^{x, y, z}=I_{2 \times 2} \otimes \cdots \otimes I_{2 \times 2} \otimes \sigma^{x, y, z} \otimes I_{2 \times 2} \otimes \cdots \otimes I_{2 \times 2},
$$

where the factor $\sigma^{x, y, z}$ occupies the $i$ th position among the $N$ factors and $\sigma^{x, y, z}$ is either of the single spin Pauli operators

$$
\sigma^{x}=\left(\begin{array}{ll}
0 & 1 \\
1 & 0
\end{array}\right), \quad \sigma^{y}=\left(\begin{array}{cc}
0 & -l \\
l & 0
\end{array}\right), \quad \sigma^{z}=\left(\begin{array}{cc}
1 & 0 \\
0 & -1
\end{array}\right) .
$$

The system Hilbert space $\mathcal{H}$ on which $H$ acts is conveniently taken as $\mathbb{C}^{2^{N}}$. We can abstract the network of spins as a graph $\mathcal{G}=(\mathcal{V}, \mathcal{E})$, where the vertices represent the spins and the edges indicate the presence of couplings.

A particular configuration considered in this paper is that of spin rings, i.e., spin networks defined by a circular arrangement of spins, described by a $J$-coupling matrix that is circulant with nearest-neighbor coupling: 


$$
H=\sum_{i=1}^{N-1} J_{i, i+1}\left(\sigma_{i}^{x} \sigma_{i+1}^{x}+\sigma_{i}^{y} \sigma_{i+1}^{y}+\eta \sigma_{i}^{z} \sigma_{i+1}^{z}\right)+J_{N, 1}\left(\sigma_{N}^{x} \sigma_{1}^{x}+\sigma_{N}^{y} \sigma_{1}^{y}+\eta \sigma_{N}^{z} \sigma_{1}^{z}\right) .
$$

The term $J_{N, 1}$ represents the coupling energy between the two ends, spins 1 and $N$, closing the ring. For networks with uniform couplings, i.e., all nonzero couplings have equal strength $J$ (in units of $\mathrm{Hz}$ ), we can set $J=1$ by choosing time in units of $J^{-1}$.

\subsection{Single excitation subspace}

Although many of the results in the following sections are more widely applicable, we primarily concern ourselves here with the single excitation subspace of the network [5], spanned by the $N$ single excitation quantum states $\{|i\rangle: i=1, \ldots, N\}$, where $|i\rangle=|\uparrow \uparrow \cdots \uparrow \downarrow \uparrow \cdots \uparrow\rangle$ with $\downarrow$ in the $i$ th position indicating that spin $i$ carries the excitation. The natural coupling among the spins allows the excitation at $i$ to drift toward an excitation at $j$ with an information transfer fidelity (ITF) that can be quantified by the maximum transition probability $p_{\max }(i, j)$. This concept will be precisely defined in the next section, but in this introductory exposition, we could think of "maximum" as the process of giving the transition from spin $i$ to $j$ the correct amount of time so that it is most likely to occur. The concepts behind these ideas are lying at the foundation of quantum mechanics as embodied in the Feynman path integral. These concepts reveal that contrary to classical least-cost-path routing that follows a single path from a source to a destination in a classical network, quantum networks follow all possible paths from the state $|i\rangle$ to the state $|j\rangle$.

\subsection{Eigendecomposition of the Hamiltonian}

Restricted to the single excitation subspace $\overline{\mathcal{H}} \cong \mathbb{C}^{N}$, the eigen decomposition of the Hamiltonian reads $\bar{H}=\sum_{k} \lambda_{k} \Pi_{k}$, where $\lambda_{k}$ for $k=1, \ldots, \widetilde{N} \leq N$ are the distinct real eigenvalues and $\Pi_{k}$ are the projectors onto the corresponding eigenspaces.

For a spin ring of size $N$ with uniform XX coupling between adjacent spins, $J_{i j}=J$ for $i=j \pm 1,(i, j)=(1, N),(i, j)=(N, 1)$, and $J_{i j}=0$ otherwise. In this case, the single excitation subspace Hamiltonian in the basis $|i\rangle=(0,0, \ldots, 0,1,0, \ldots, 0)^{T}$ becomes the circulant matrix

$$
\bar{H}_{N}=\left(\begin{array}{ccccccc}
0 & 1 & 0 & \ldots & & 0 & 1 \\
1 & 0 & 1 & & & 0 & 0 \\
0 & 1 & 0 & \ddots & & 0 & 0 \\
\vdots & \ddots & & & & \vdots \\
& & & & & \ddots & \\
0 & 0 & 0 & & \ddots & 0 & 1 \\
1 & 0 & 0 & \ldots & & 1 & 0
\end{array}\right)\left(=: C_{N}\right)
$$


Table 1 Eigenvalues and eigenvectors of Hamiltonian $\bar{H}$ over single excitation subspace [15] in the basis where $|i\rangle=e_{i}:=\left(\begin{array}{lllll}0 & \ldots & 0 & 0 & \ldots\end{array}\right)^{T} \cdot \rho_{N}=\exp (2 \pi \iota / N)$ and $\left|v_{k}\right\rangle_{j}$ denotes the $j$ th component of $\left|v_{k}\right\rangle$

\begin{tabular}{lll}
\hline & $\lambda_{k=0, \ldots, N-1}$ & $\left|v_{k}\right\rangle_{j=0, \ldots, N-1}$ \\
\hline XX coupling $(\eta=0)$ & $2 \cos \left(\frac{2 \pi k}{N}\right)$ & $\sqrt{\frac{1}{N}} \rho_{N}^{k(j-1)}$ \\
Heisenberg coupling $(\eta=1)$ & $2 \cos \left(\frac{2 \pi k}{N}\right)+1$ & $\sqrt{\frac{1}{N}} \rho_{N}^{k(j-1)}$ \\
\hline
\end{tabular}

where the subscript $N$ is utilized to indicate that the system has $N$ spins. For uniform Heisenberg coupling, the Hamiltonian is the same except for the addition of a multiple of the identity, which simply shifts the eigenvalues by a constant and does not affect the eigenvector structure or differences between eigenvalues. The eigenvalues and eigenvectors of circulant matrices are well known and shown in Table 1 . The $N$ single excitation eigenvalues are conveniently parameterized by an integer $k$ running from 0 to $N-1$ or 1 to $N$ with the cyclic condition that $\lambda_{0}=\lambda_{N}$.

The following lemma regarding the eigenvalues will be helpful later.

Lemma 1 For a spin ring of size $N$ with uniform $X X$ couplings, we have:

- For $N$ even, but not divisible by 4 , then the spectrum of $\bar{H}_{N}$ has mirror symmetry relative to the origin; precisely, we have $\lambda_{k}=\lambda_{N-k}=-\lambda_{N / 2-k}=-\lambda_{N / 2+k} \neq$ 0 , i.e., there are $\frac{1}{2} N-1$ distinct pairs of double eigenvalues, and two single eigenvalues \pm 2 , giving a total of $\widetilde{N}=(N+2) / 2$ pairwise distinct eigenvalues:

$$
\left\{-2, \lambda_{k}, 2: k=1, \ldots, \frac{1}{2} N-1\right\} .
$$

If $N$ is divisible by 4 , then the spectrum has a total of $\widetilde{N}=(N+2) / 2$ pairwise distinct eigenvalues and a double eigenvalue at $0\left(\right.$ for $\left.k=\frac{1}{4} N, \frac{3}{4} N\right)$.

- For $N$ odd, we have $\lambda_{N-k}=\lambda_{k} \neq 0$ and there are $(N-1) / 2$ distinct pairs of double eigenvalues and a single eigenvalue +2 , giving a total of $\widetilde{N}:=(N+1) / 2$ distinct eigenvalues:

$$
\left\{\lambda_{k},+2: k=1, \ldots, \frac{1}{2}(N-1)\right\}
$$

- In either case, the number of pairwise distinct eigenvalues is

$$
\widetilde{N}:=\left\lceil\frac{N-1}{2}\right\rceil+1=\left\lceil\frac{N+1}{2}\right\rceil
$$

Moreover, the eigenvalues of $C_{N}$ and $C_{N-1}$ are interlaced.

Proof The listed items are trivial. The last claim is the Cauchy interlacing property [16].

For a double eigenvalue $\lambda_{k}=\lambda_{N-k}$, denote the projection on the corresponding eigenspace as $\Pi_{k}:=\left|v_{k}\right\rangle\left\langle v_{k}|+| v_{N-k}\right\rangle\left\langle v_{N-k}\right|$, where the eigenvectors can be chosen 
such that $v_{N-k}=v_{k}^{*}$. Moreover, for the single eigenvalue $\lambda_{0}=+2$, define $\Pi_{0}:=$ $\left|v_{0}\right\rangle\left\langle v_{0}\right|$ to be its eigenprojection. If $N$ is even, the single eigenvalue $\lambda_{N / 2}=-2$ has its eigenprojection denoted as $\Pi_{N / 2}:=\left|v_{N / 2}\right\rangle\left\langle v_{N / 2}\right|$. If, in addition, $N$ is divisible by 4 , denote the eigenprojection of the double eigenvalue $\lambda_{N / 4}=\lambda_{3 N / 4}=0$ as $\Pi_{N / 4}:=\left|v_{N / 4}\right\rangle\left\langle v_{N / 4}|+| v_{3 N / 4}\right\rangle\left\langle v_{3 N / 4}\right|$. With this notation, the Hamiltonian restricted to the single excitation subspace can be written as

$$
\bar{H}=\sum_{k=0}^{\tilde{N}-1} \lambda_{k} \Pi_{k} .
$$

The above can easily be extended to the Heisenberg case by globally shifting the eigenvalues by 1 .

\section{Maximum transfer fidelity and attainability}

Let $|i\rangle \in \overline{\mathcal{H}}$ be a quantum state with excitation localized at spin $i$. The quantum mechanical probability of transition from state $|i\rangle$ to state $|j\rangle$ in an amount of time $t$ is given by

$$
p_{t}(i, j)=\left|\left\langle i\left|\mathrm{e}^{-i \hbar H_{1} t}\right| j\right\rangle\right|^{2},
$$

where we choose energies in units of $\hbar / J$ allowing us to assume $\hbar=1$ and omit $\hbar$ in the following. This formula is a corollary of the Feynman path integral $[17,18]$. To circumvent the difficulty posed by the time dependence of this probability, we proceed as in [13] and define the maximum transition probability $p_{\max }(i, j)$ also referred to as information transfer fidelity $(I T F)$ :

$$
\begin{aligned}
p_{t}(i, j) & =\left|\left\langle i\left|\mathrm{e}^{-\imath H_{1} t}\right| j\right\rangle\right|^{2}=\left|\sum_{k=0}^{\widetilde{N}-1}\left\langle i\left|\Pi_{k}\right| j\right\rangle \mathrm{e}^{-\imath \lambda_{k} t}\right|^{2} \\
& \leq\left(\sum_{k=0}^{\widetilde{N}-1}\left|\left\langle i\left|\Pi_{k}\right| j\right\rangle\right|\right)^{2}=: p_{\max }(i, j)
\end{aligned}
$$

Clearly, $p_{\max }(i, j) \leq 1$. Observe that instead of taking the sum of the absolute values of all $\left\langle i\left|\Pi_{k}\right| j\right\rangle$ terms, we could take the sum of the absolute values of some partial sums of such terms and derive other upper bounds. Note that the upper bound is valid for any spin network, no matter how many spins, no matter how many multiple eigenvalues, no matter the topology. Since the upper bound depends only on the eigenvectors of the Hamiltonian and since those are continuously dependent on the strengths of the couplings, the upper bound is continuous relative to the $J_{i j}$.

\subsection{Attainability of bounds}

The ITF $p_{\max }(i, j)$ is an upper bound on $p_{t}(i, j)$, which acquires its full significance if $p_{\text {max }}$ is achievable, that is, if there exists a sequence of time samples $\left\{t_{i, j}(n): n \in \mathbb{N}\right\}$ 
such that $\lim _{n \rightarrow \infty} p_{t_{i j}(n)}(i, j)=p_{\max }(i, j)$. Observing that the absolute value in Eq. (4) will absorb any global phase factor, the attainability condition is that there exists $t \in[0, \infty)$ such that

$$
\mathrm{e}^{-l \lambda_{k} t}=s_{k}(i, j) \mathrm{e}^{l \phi}, \quad \forall k=0, \ldots, \tilde{N}-1
$$

where $s_{k}(i, j):=\operatorname{Sgn}\left(\left\langle i|| \Pi_{k} \mid j\right\rangle\right) \in\{0, \pm 1\}$ is a sign factor and $\phi$ is a global phase, which is arbitrary but must be the same for all $k$ 's. Eigenspaces with $s_{k}=0$ (where the $(i, j)$ dependency is suppressed to avoid the clutter) have no overlap with the initial and/or target state and do not contribute to the sum. We shall refer to them as dark-state subspaces. They can be ignored, and we can restrict ourselves to the set $K^{\prime} \subseteq\{0,1, \ldots, \widetilde{N}-1\}$ of indices $k$ for which $s_{k} \neq 0$. The physical interpretation of $K^{\prime}$ is the set of eigenspaces $\Pi_{k} \overline{\mathcal{H}}$ that have nontrivial overlap with the initial and target states. Noting that $s_{k}= \pm 1$ for $k \in K^{\prime}$, and $\exp \left[-l \frac{\pi}{2}\left(s_{k}-1\right)\right]=1$ for $s_{k}=1$ and $\exp \left[-\imath \frac{\pi}{2}\left(s_{k}-1\right)\right]=-1$ for $s_{k}=-1$, we can write

$$
s_{k}=\exp \left[-\imath \pi\left(2 n_{k}+\frac{1}{2}\left(s_{k}-1\right)\right)\right], \quad \forall k \in K^{\prime},
$$

where $n_{k} \in \mathbb{Z}$ is an arbitrary integer. Inserting this into (5), taking the logarithm and dividing by $-l$ yield

$$
\lambda_{k} t=2 \pi n_{k}+\frac{\pi}{2}\left(s_{k}-1\right)-\phi, \quad \forall k \in K^{\prime}
$$

This condition is not directly useful as $\phi$ can be arbitrary, but we obtain meaningful constraints if we subtract the equations in a pairwise manner, with $k \neq \ell$ :

$$
\left(\lambda_{k}-\lambda_{\ell}\right) t=2 \pi\left(n_{k}-n_{\ell}\right)+\frac{\pi}{2}\left(s_{k}-s_{\ell}\right), \quad \forall k, \ell \in K^{\prime}
$$

We can also write the attainability constraints more explicitly:

$$
\begin{aligned}
& \left(\lambda_{k}-\lambda_{\ell}\right) t=2 \pi\left(n_{k}-n_{\ell}\right), \quad \text { if } s_{\ell}=s_{k}, \\
& \left(\lambda_{k}-\lambda_{\ell}\right) t=2 \pi\left(n_{k}-n_{\ell}\right)+\pi, \quad \text { if } s_{k}=-s_{\ell}=1 \text {, } \\
& \left(\lambda_{k}-\lambda_{\ell}\right) t=2 \pi\left(n_{k}-n_{\ell}\right)-\pi, \quad \text { if } s_{k}=-s_{\ell}=-1 \text {. }
\end{aligned}
$$

These conditions are necessary and sufficient for attainability. They are physical, only involving differences of the eigenvalues, which are observable and independent of arbitrary phases. Vanishing left-hand sides in the above are not an issue, as we are only looking at the differences, which are nonzero by definition as $\lambda_{k}, k \in K^{\prime}$, are the distinct eigenvalues of $\bar{H}$.

Observe that all of the equations are compatible. Indeed, adding Eq. (8) for $(k, \ell)$ and $(\ell, m)$ yields $(8)$ for $(k, m)$. Naturally, these equations are redundant, but we obtain a set of linearly independent equations if we exclude the dark-state subspaces and restrict ourselves to a suitable subset of equations, e.g., $\left(k_{i-1}, k_{i}\right)$ or $\left(k_{0}, k_{i}\right)$ for $K^{\prime}=\left\{k_{1}^{\prime}, k_{2}^{\prime}, \ldots, K_{\bar{N}}^{\prime}\right\}$. 
Example 1 (Dark States for Rings.) For ring systems with uniform XX coupling, the distinct eigenvalues are $\lambda_{k}=2 \cos (2 \pi k / N)$. For eigenvalues of multiplicity 1 , which occur for $k=0$, and $k=\frac{1}{2} N$ if $N$ is even, $\left\langle i\left|\Pi_{0}\right| j\right\rangle=(1 / N) \neq 0$ and $\left\langle i\left|\Pi_{N / 2}\right| j\right\rangle=(1 / N)(-1)^{i-j} \neq 0$; therefore, there are no dark states associated with these eigenvalues. For eigenvalues with multiplicity $2,\left\langle i\left|\Pi_{k}\right| j\right\rangle=\frac{2}{N} \cos \left(\frac{\pi}{2} n\right)$ with $n=4 k(i-j) / N$ for $k=0, \ldots, \Gamma(N-4) / 2\rceil$; therefore, there are dark states if and only if $n$ is an odd integer. This can happen only if $N$ is divisible by 4 . The same holds of rings with uniform Heisenberg coupling as they have the same eigenspace structure and the differences between eigenvalues are the same.

\subsection{Simultaneous attainability and flows on the torus}

Excluding dark-state subspaces, restricting (8) to a subset $\mathcal{S} \subseteq K^{\prime} \times K^{\prime}$ of linearly independent equations, and setting $\omega_{k, \ell}=\left(\lambda_{k}-\lambda_{\ell}\right) / \pi$, the attainability conditions become

$$
t \omega_{k, \ell}=\frac{1}{2}\left(s_{k}-s_{\ell}\right) \quad \bmod 2, \quad \omega_{k, \ell}:=\left(\lambda_{k}-\lambda_{\ell}\right) / \pi, \quad \forall(k, \ell) \in \mathcal{S} .
$$

The left-hand side of the above is the solution of the flow on the torus $\dot{x}=\omega_{k \ell}$, with $x(0)=0$. In this dynamic formulation, the question is whether the flow starting at $x(0)=0$ passes through the point with coordinates 0 or 1 , depending on whether $s_{k}=s_{\ell}$ or $s_{k} \neq s_{\ell}$, respectively. It is well known [19, Prop.1.5.1] that the flow starting at an arbitrary $x(0)$ (which includes $x(0)=0$ ) passes arbitrarily close to an arbitrary point on the torus if and only if the $\omega_{k, \ell}$ 's are linearly independent over the rationals $\mathbb{Q}$. This property of the flow getting arbitrarily close to an arbitrary point from an arbitrary initial condition is very strong and referred to as minimality. Observe that for the flow to be minimal it suffices that starting at $x(0)=0$, it gets arbitrarily close to any point. Obviously, minimality is sufficient but not necessary for attainability, as the latter only requires the flow to pass arbitrarily close to a specific point on the torus, while minimality guarantees that the flow can get arbitrarily close to any point.

Recall that Eq. (8) refers to a specific but arbitrary transfer $|i\rangle \rightarrow|j\rangle$, as the signs depend on $i, j$. We could consider all Eq. (8)'s for all $i \neq j$ and ask the question as to whether there exists a unique $t$ such that attainability holds for all $i \neq j$. We refer to this stronger version of attainability as simultaneous attainability.

If for a given pair $(i, j)$ there are at least three nondark eigenspaces corresponding to $s_{d}, s_{m}, s_{n} \in\{ \pm 1\}$, then there must exist a pair, say $(m, n)$, with $s_{m}-s_{n}=0$. In this case, setting $t=2 \tau / \omega_{m n}$ for $\tau \in \mathbb{N}$ ensures that the $(m, n)$ Eq. (9) holds exactly and the remaining attainability equations become

$$
\theta_{k \ell} \tau=\frac{1}{2}\left(s_{k}-s_{\ell}\right) \quad \bmod 2, \quad \theta_{k \ell}:=2 \omega_{k \ell} / \omega_{m, n}, \quad \forall(k, \ell) \in \mathcal{S}_{0}:=\mathcal{S} \backslash\{(m, n)\} .
$$

The left-hand side $\theta_{k \ell} \tau$ of the preceding equation is the solution of the translation on the torus, that is, $x(\tau+1)=x(\tau)+\theta_{k \ell} \bmod 2$ with initial condition $x(0)=0$. By [19, Prop.1.4.1], the translation on the torus can come arbitrarily close to any point iff the elements in the set $\{1\} \cup\left\{\theta_{k \ell}:(k, \ell) \in \mathcal{S}_{0}\right\}$ are linearly independent over $\mathbb{Q}$. As 
before, the linear independence is sufficient, but not necessary for attainability. Note that we can in principle always reorder the eigenvalues so that the reference transition is $(m, n)=(1,2)$.

It should be noted that the attainability criteria above apply to any spin network. For specific types of networks, we can derive more explicit criteria.

Example 2 (Attainability Condition for Rings.) Given the formula for the eigenvalues for homogeneous rings, $\lambda_{k}=2 \cos (2 \pi k / N)$, elementary trigonometry shows that

$$
\omega_{k \ell}=\frac{1}{\pi}\left(\lambda_{k}-\lambda_{\ell}\right)=-\frac{4}{\pi} \sin \left(\frac{\pi}{N}(k+\ell)\right) \sin \left(\frac{\pi}{N}(k-\ell)\right) .
$$

There are $\tilde{N}=\lfloor N / 2\rfloor+1$ eigenspaces and $\widetilde{N}-1$ independent transition frequencies $\omega_{k \ell}$. Choosing the subset of linearly independent equations $\mathcal{S}=\{(k, k+1): k=$ $0, \ldots, \bar{N}\}, \bar{N}:=\widetilde{N}-2$, with the ordering of the eigenspaces as defined above, the attainability conditions can be written as

$$
\frac{4}{\pi} \sin \left(\frac{\pi}{N}(2 k+1)\right) \sin \left(\frac{\pi}{N}\right)=\frac{1}{2}\left(s_{k}-s_{k+1}\right) \quad \bmod 2, \quad \forall k=0, \ldots, \bar{N}
$$

If $s_{m}=s_{m+1}$, then setting $t=2 \tau / \omega_{m, m+1}$ for $\tau \in \mathbb{N}$ ensures $\omega_{m, m+1} t=2 \tau=0$ $\bmod 2$ and the attainability conditions become

$$
\theta_{k} \tau=\frac{1}{2}\left(s_{k}-s_{k+1}\right) \quad \bmod 2, \quad \forall k=0, \ldots, \bar{N}
$$

with $\theta_{k}=\sin \left(\frac{\pi}{N}(2 k+1)\right) / \sin \left(\frac{\pi}{N}(2 m+1)\right)$. Notice that the signs of the projections of the initial state $|i\rangle$ and target state $|j\rangle, s_{k}=\left\langle j\left|\Pi_{k}\right| i\right\rangle$, depend on the choices of the latter, and it may happen that the signs $s_{k}$ are alternating, $s_{k+1}=-s_{k}$ for all $k$. In this case, the problem can easily be rectified by reordering the eigenvalues, e.g., so that $s_{0}^{\prime}=s_{1}^{\prime}$ with the new ordering.

Example 3 (Rational Independence.) Applying the previous results to a ring of $N=5$ spins, the number of pairwise distinct eigenvalues of the single excitation Hamiltonian $\bar{H}$ is $\widetilde{N}=3$ and there are two linearly independent transition frequencies $\omega_{01}=-\frac{4}{\pi} \sin \left(\frac{1}{5} \pi\right) \sin \left(\frac{1}{5} \pi\right)$ and $\omega_{12}=-\frac{4}{\pi} \sin \left(\frac{3}{5} \pi\right) \sin \left(\pi \frac{1}{5}\right)$. To verify linear independence of $\left\{\sin \left(\frac{1}{5} \pi\right), \sin \left(\frac{3}{5} \pi\right)\right\}$ over $\mathbb{Q}$, we must show that the equation

$$
\alpha_{1} \sin \left(\frac{\pi}{5}\right)+\alpha_{3} \sin \left(\frac{3 \pi}{5}\right)=0 \text { for } \alpha_{1}, \alpha_{3} \in \mathbb{Q},
$$

has only the trivial solution $\alpha_{1}=\alpha_{3}=0$ over $\mathbb{Q}$. Using $\sin \left(\frac{\pi}{5}\right)=\frac{1}{4} \sqrt{10-2 \sqrt{5}}$ and $\sin \left(\frac{3 \pi}{5}\right)=\frac{1}{4} \sqrt{10+2 \sqrt{5}}$, we can rewrite the equation as $\alpha_{1}^{2}(10-2 \sqrt{5})=$ $\alpha_{3}^{2}(10+2 \sqrt{5})$. Viewing the field $\mathbb{Q}(\sqrt{5})$ as a two-dimensional vector space over $\mathbb{Q}$ with basis $1, \sqrt{5}$ gives two equations $\alpha_{1}^{2}=\alpha_{3}^{2}$ and $\alpha_{1}^{2}=-\alpha_{3}^{2}$, which much be simultaneously satisfied. This is possible only for $\alpha_{1}=\alpha_{3}=0$. Thus, the flow on the torus is minimal, and $p_{\max }(i, j)$ is attainable for all $(i, j)$.

Example 4 (Rational Dependence for Even Rings.) For a ring with $N=10$ spins, there are $\widetilde{N}=6$ distinct eigenvalues and five primary transition frequencies $\omega_{k, k+1}$ 
for $k=0, \ldots, 4$. Noting that $\sin \left(\frac{5 \pi}{10}\right)=1, \sin \left(\frac{\pi}{10}\right)=\sin \left(\frac{9 \pi}{10}\right)=\frac{1}{4}(-1+\sqrt{5})$ and $\sin \left(\frac{3 \pi}{10}\right)=\sin \left(\frac{7 \pi}{10}\right)=\frac{1}{4}(1+\sqrt{5})$. It is easily seen that $\alpha=(2,-2,1)$ is a $\mathbb{Q}$-solution to the linear dependence equation

$$
\alpha_{1} \sin \left(\frac{9 \pi}{10}\right)+\alpha_{2} \sin \left(\frac{3 \pi}{10}\right)+4 \alpha_{3} \sin \left(\frac{5 \pi}{10}\right)=0
$$

Hence, the $p_{\max }(i, j)$ are not simultaneously attainable—although $p_{\max }(i, j)$ may be attainable for some $(i, j)$.

More generally, for a ring with $N$ even, there are $\frac{1}{2} N$ transition frequencies

$$
\omega_{k, k+1}=\frac{4}{\pi} \sin \left((2 k+1) \frac{\pi}{N}\right) \sin \left(\frac{\pi}{N}\right)
$$

which occur in pairs $\omega_{k, k+1}=\omega_{\bar{N}-k, \bar{N}-k+1}$ with $\bar{N}=\frac{1}{2} N-1$, precluding rational independence.

Example 5 (Rational Dependence for Odd Rings.) Similarly, we can easily verify that for a ring with $N=9$ spins, the transition frequencies are not rationally independent, as we have, e.g., $\sin (7 \pi / 9)-\sin (5 \pi / 9)+\sin (\pi / 9)=0$ and thus $\omega_{3,4}-\omega_{2,3}+\omega_{0,1}=0$.

In general, rational independence of the transition frequencies for homogeneous rings does not hold when $N$ is not prime.

\subsection{Simultaneous Diophantine approximation}

Instead of checking rational independence of $\{1\} \cup\left\{\theta_{k \ell}:(k, \ell) \in \mathcal{S}_{0}\right\}$, a less conservative approach is to proceed, either analytically or computationally [20,21], via the simultaneous Diophantine approximation [22-25] by finding integers $p_{k \ell}, q$ such that

$$
\left|\theta_{k \ell}-\frac{p_{k \ell}}{q}\right| \leq \frac{c}{q^{1+\epsilon}}, \quad \forall(k, \ell) \in \mathcal{S}_{0},
$$

and $\epsilon>0$. With $\tau=q$, the above yields

$$
\left|\theta_{k \ell} \tau-p_{k \ell}\right| \leq \frac{c}{\tau^{\epsilon}}, \quad \forall(k, \ell) \in \mathcal{S}_{0}
$$

In the single-dimensional case, the solution is well known to be given by the continued fraction expansion of $\theta$. Truncating the continued fraction expansion yields convergents, i.e., rational fractions $p / q$, with errors bounded as $|\theta q-p| \leq 1 / q$, which is optimal among all rational approximations of denominators less than or equal to $q$. The major hurdle at extending this result to the multi-dimensional case is that there is an incompatibility between the unimodular property of the multi-dimensional continued fraction (MCF) solution and optimality. 
Nevertheless, the celebrated Dirichlet box principle shows that there are multidimensional approximations with $c=1$ and $\epsilon=1 / \bar{N}$, where in the present context $\bar{N}=\left|\mathcal{S}_{0}\right|$. Moreover, there are infinitely many integer solutions $q$ to the simultaneous Diophantine approximation; in other words, as $\tau$ is allowed to become arbitrarily large, the above error can be made arbitrarily small. The constant $c=1$ can hardly be improved as for $c<1$ there are "badly approximable vectors" $\theta \in \mathbb{R}^{\bar{N}}$ defined by $\liminf _{q \rightarrow \infty} q^{1 / \bar{N}} d\left(\theta q, \mathbb{Z}^{\bar{N}}\right)>0$ such that the simultaneous Diophantine approximation has only finitely many solutions [22,26, Sec. 5]. If, however, $c$ is allowed to depend on $\bar{N}$, refined bounds $(c<1)$ can be derived on $c(\bar{N})$ due to the existence of infinitely many solutions [23]. Specializing the approximation to $\bar{N}=2$, it can be shown [27] that the bound can be improved down to $c=8 / 13$, along with $\epsilon=1 / 2$. In the one-dimensional case Hurwitz's theorem says that one can take $c=1 / \sqrt{5}$ and $\epsilon=1$. On a general tone, the Dirichlet approximation can only be improved slightly and at the expense of considerable extra difficulties; we will therefore work exclusively with the Dirichlet approximation in the following.

Assuming we have obtained a Dirichlet-good simultaneous Diophantine approximation, the approximate attainability conditions become

$$
p_{k \ell}=\frac{1}{2}\left(s_{k}-s_{\ell}\right) \quad \bmod 2, \quad \forall(k, \ell) \in \mathcal{S}_{0}
$$

The difficulty is to find, if it exists, a simultaneous Diophantine approximation of Dirichlet accuracy that satisfies the above conditions on the numerators. The following example demonstrates that it is not, in general, possible to achieve the even/odd conditions (12) on the numerators $p_{k \ell}$ without compromising on the accuracy of the Diophantine approximation. To be more specific, arbitrary accuracy can still be achieved with Conditions (12), but a larger denominator is required to achieve the same level of accuracy in the presence of the constraints.

Example 6 (Simultaneous Diophantine Approximation with Constraints.) In Example 3, the flow on the torus for a ring with $N=5$ was found to be minimal, implying that we can get arbitrarily close to an arbitrary point on the torus. By the preceding argument, this guarantees existence of simultaneous Diophantine approximations of arbitrary accuracies with prescribed even/odd numerators. Furthermore, it is readily found that

$$
\theta_{12}=\frac{2 \sin (3 \pi / 5)}{\sin (\pi / 5)}=1+\sqrt{5}=[3 ; 4,4,4,4,4, \ldots],
$$

where the final expression denotes the continued fraction expansion giving the optimal rational approximations [28, Chap. 10]. It is known that quadratic irrationality leads to continued fractions that eventually stabilize. The first convergents are

$$
3, \frac{13}{4}, \frac{55}{17}, \frac{233}{72}, \frac{987}{305}, \frac{4181}{1292}, \frac{17711}{5473}, \frac{75025}{23184}, \frac{317811}{98209}, \frac{1346269}{416020}, \ldots
$$

Observe that all of them have odd numerators, while the approximations we require must have even numerators since for the $N=5$ ring $s_{0}=s_{1}=1$. This can be rectified 
by using the so-called semi-convergents [29, Sec. V.4], [21]. Given two convergents ordered as $p_{n-1} / q_{n-1}<p_{n} / q_{n}$, one can easily squeeze a semi-convergent between them as follows:

$$
\frac{p_{n-1}}{q_{n-1}}<\frac{p_{n-1}+p_{n}}{q_{n-1}+q_{n}}<\frac{p_{n}}{q_{n}} .
$$

The semi-convergent has even numerator and has the accuracy of the convergents $p_{n-1} / q_{n-1}$ and $p_{n} / q_{n}$ but at the cost of doubling the denominator. To prove that the semi-convergents provide approximations of arbitrary accuracy, it suffices to show that there are infinitely many $n$ 's such that $p_{n-1} / q_{n-1}<p_{n} / q_{n}$. This is a corollary of the unimodular property of continuous fractions, saying that $p_{n-1} q_{n}-p_{n} q_{n-1}$ is alternately \pm 1 .

We propose a general iterative method to deal with the even/odd constraints. To simplify the notation, let $\theta \in \mathbb{R}^{\bar{N}}, p \in \mathbb{Z}^{\bar{N}}$, be column vectorizations of the $\theta_{k \ell} \mathrm{s}$, $p_{k \ell}$ s, respectively, where $\bar{N}:=\left|\mathcal{S}_{0}\right|$. We want to come up with a Dirichlet-good approximation, $\theta \approx p / q$, where $p \in \mathbb{Z}^{\bar{N}}, q \in \mathbb{N}$, with even/odd constraints on the numerators $p_{i}$. By "Dirichlet-good," we mean that the infinity error is bounded as $\|\theta q-p\|_{\infty} \leq c / q^{1 / \bar{N}}$, where $c$ is a constant independent of $\bar{N}$ and $q$. The idea is to iteratively scale $\theta$ by (the inverse of) a diagonal matrix of positive rational numbers, $\bar{\theta}=Y(n)^{-1} \theta$, compute a Dirichlet-good approximation of $\bar{\theta}$ using, e.g., the Dirichlet box principle, or the LLL-algorithm, or Lagarias' multi-dimensional continued fractions (MCFs), and then revise the scaling to meet the even/odd constraints, with the hope that the procedure will converge. Write the Dirichlet-good approximation $\bar{\theta} \approx \bar{p} / q$ and manipulate it as follows:

$$
\begin{gathered}
\|\bar{\theta} q-\bar{p}\|_{\infty} \quad \leq \frac{1}{q^{1 / \bar{N}}} \\
\min _{i}\left(Y(n)_{i i}^{-1}\right)\|\theta q-Y(n) \bar{p}\|_{\infty} \leq\left\|Y(n)^{-1}(\theta q-Y(n) \bar{p})\right\|_{\infty} \leq \frac{1}{q^{1 / N}}
\end{gathered}
$$

It follows that

$$
\|\theta q-Y(n) \bar{p}(Y(n))\|_{\infty} \leq \frac{1}{q^{1 / \bar{N}}} \max _{i} Y(n)_{i i} .
$$

In other words, $Y(n) \bar{p} / q$ is a Dirichlet-good approximation of $\theta$, $\operatorname{provided}^{\max _{i}} Y(n)_{i i}$ can be dominated by a bound independent of $q$ and $\bar{N}$. Because the initial choice of $Y(n)$ is arbitrary, it is not guaranteed that $Y(n) \bar{p}$ has the correct even/odd property. Nevertheless, we could revise $Y(n)$ to meet those properties. If a component $\bar{p}_{i}$ comes out to be odd and needs to be even, we choose $Y(n+1)_{i i}=2$. If the algorithm has converged, that is $Y(n+1)=Y(n)$, then the bound becomes

$$
\|\theta q-Y(n+1) \bar{p}(Y(n))\|_{\infty} \leq 2 \frac{1}{q^{1 / \bar{N}}} .
$$

Conversely, if $\bar{p}_{i}$ comes out to be even with $2^{d}$ in its prime number decomposition, we take $Y(n+1)_{i i}=1 / 2^{d}$ and, at convergence, the bound on the $i$ th component 
becomes

$$
\left|\theta_{i} q-2^{-d} \bar{p}_{i}\right| \leq 2^{-d} \frac{1}{q^{1 / \bar{N}}}
$$

Then this procedure is repeated with the scaling $\bar{\theta}=Y(n+1) \theta$, in the hope that it converges.

Theorem 1 Given $\theta \in \mathbb{R}^{\bar{N}}$, assuming $\{Y(n)\}$ converges, there exists a simultaneous Diophantine approximation $\theta \approx p / q$ satisfying prescribed even/odd constraints on the numerators $p_{i}, i=1, \ldots, \bar{N}$, with an error bound $\|\theta q-p\|_{\infty} \leq 2 / q^{1 / \bar{N}}$ that is off the usual Dirichlet bound by a factor not exceeding 2.

Example 7 (Simultaneous Diophantine Approximation with Constraints.) We consider the same situation as in Example 6, where all convergents of $\theta_{12}$ have odd numerators while attainability calls for an even numerator. We initiate the algorithm with $Y(0)=1$, that is, $\bar{\theta}=\theta=1+\sqrt{5}$. Whatever convergent $\bar{p} / q$ we pick, it has odd numerator, and hence we take $Y(1)=2$. We hence rewrite the continued fraction decomposition with $\bar{\theta}=(1 / 2) \theta=(1+\sqrt{5}) / 2$, which gives the convergents

$$
1,2, \frac{3}{2}, \frac{5}{3}, \frac{8}{5}, \frac{13}{8}, \frac{21}{13}, \frac{34}{21}, \frac{55}{34}, \frac{89}{55}, \frac{144}{89}, \frac{233}{144}, \frac{377}{233}, \frac{610}{377}, \frac{987}{610}, \ldots
$$

To secure convergence, $Y(3)=Y(2)=2$, we need to pick a convergent with odd numerator, say, 377/233, and the new Diophantine approximation of $\theta_{12}$ is $2 \times 377 / 233$. This gives an error $\left|\theta_{12} \times 233-754\right|=0.0038<2 / 233=0.0086$, as claimed.

\section{4 (Weighted) LLL-algorithm}

Even though Theorem 1 guarantees that under convergence conditions, Dirichletgood simultaneous Diophantine approximations can be manipulated so as to yield numerators that have prescribed even/odd properties, we are still left with the problem of coming up with simultaneous Diophantine approximations in the first place.

One of the first computational solutions to the simultaneous Diophantine approximation was the so-called LLL-algorithm by Lenstra, Lenstra and Lovász [21,24,30]. An alternative algorithm based on geodesic multi-dimensional continued fraction expansion was proposed by Lagarias [31]. Both approaches proceed by reduction in the basis of the lattice generated by the columns of

$$
B(\mathrm{~s})=\left(\begin{array}{cc}
I_{\bar{N} \times \bar{N}} & -\theta \\
0_{1 \times \bar{N}} & \mathrm{~s}
\end{array}\right),
$$

where $\mathbf{\downarrow} 0$ is a scaling parameter. Observing that $B(\mathrm{~s})(p, q)^{T}=(p-\theta q, \mathrm{~s} q)^{T}$, it follows that a short vector in the lattice $B(\mathrm{~s}) \mathbb{Z}^{\bar{N}+1}$ yields a good approximation. The numerator of this good approximation could be "fixed" by the procedure of Sect. 3.3 
to satisfy the even/odd requirement. However, it is proposed to combine the two procedures into a single one-computation of a good approximation from a short lattice vector and fixing the numerator-by introducing a nonuniform diagonal scaling and work on the lattice $\Lambda(\mathrm{s}, X)$ generated by the columns of

$$
B(\mathrm{~s}, X)=\left(\begin{array}{cc}
X & -X \theta \\
0_{1 \times \bar{N}} & \mathrm{~s}
\end{array}\right)
$$

where $X=\operatorname{diag}\left(x_{1}, \ldots, x_{\bar{N}}\right)$. Note that for $\mathrm{s}=1$ and $X=x I_{\bar{N} \times \bar{N}}$, we recover the scaling of [21]. Like the algorithm of Sect. 3.3, this procedure is not guaranteed to be successful, but if it is, it yields solutions guaranteed to be optimal relative to some criterion. The LLL-algorithm produces a basis of short Euclidean norm vectors $\left(b^{*}(\mathrm{~s}, X)_{1}, b^{*}(\mathrm{~s}, X)_{2}, \ldots, b^{*}(\mathrm{~s}, X)_{\bar{N}+1}\right)=: B^{*}(\mathrm{~s}, X)$ such that

$$
\left\|b^{*}(\mathrm{~s}, X)_{1}\right\|<\left\|b^{*}(\mathrm{~s}, X)_{j}\right\|, \quad j=2, \ldots \bar{N}+1 .
$$

The $b^{*}(\mathrm{~s}, X)_{1}$ vector is very close to the shortest one. A refined version of the LLLalgorithm captures the genuinely shortest vector of the lattice $\Lambda(\mathrm{s}, X)$ as follows: Given the reduced basis $\left\{b^{*}(\mathrm{~s}, X)_{i}: i=1, \ldots, \bar{N}+1\right\}$, it can be shown that the shortest (in the sense of the Euclidean norm) lattice vector is to be sought among all lattice vectors of the form $\sum_{i} \beta_{i} b^{*}(\mathrm{~s}, X)_{i},\left|\beta_{i}\right| \leq(2 / \sqrt{3})^{\bar{N}+1}$. Lagarias' theorem [24, Lemma 5] then implies that a shortest Euclidean norm vector of the lattice is a best $X$-weighted Diophantine approximation. Observing that

$$
B(\mathrm{~s}, X)\left(\begin{array}{l}
p \\
q
\end{array}\right)=\left(\begin{array}{c}
X(p-\theta q) \\
\mathrm{s} q
\end{array}\right)
$$

and taking $\mathrm{s} \downarrow 0$, it becomes clear that a short vector in the lattice $B(\mathrm{~s}, X) \mathbb{Z}^{\bar{N}+1}$ provides a good $X$-weighted Diophantine approximation:

$$
q=\frac{\left(B^{*}(\mathrm{~s}, X)\right)_{\bar{N}+1,1}}{\mathrm{~s}}, \quad p_{i}=\frac{\left(B^{*}(\mathrm{~s}, X)\right)_{i, 1}}{x_{i}}+\theta_{i} q .
$$

With the shortest vector, we construct the best approximation, that is, the approximation that minimizes

$$
\|X(\theta q-p)\|_{2}
$$

in the same way as for the good approximation.

Before proceeding any further, we take care of a technicality: As one would expect, the simulations also suggest that $q$ grows without bound as $\mathrm{S}$ decreases to zero. For the weighted LLL-algorithm, we can prove the following: 
Theorem 2 For the weighted LLL-algorithm to solve

$$
(\hat{p}(\mathrm{~s}), \hat{q}(\mathrm{~s}))=\arg \min _{(p, q) \in \mathbb{Z}^{\bar{N}+1}}\left\|B(\mathrm{~s}, X)\left(\begin{array}{l}
p \\
q
\end{array}\right)\right\|_{X \oplus 1}
$$

where $\|\cdot\|_{X \oplus 1}$ is the Euclidean norm weighted by the direct sum of $X$ and 1 , we have $\lim _{\mathbf{s} \downarrow 0} \hat{q}(\mathrm{~s})=\infty$.

Proof Assume that there exist $\mathrm{s}_{\min }$ and $q_{\max }$ such that $\forall \mathrm{s} \leq \mathrm{s}_{\min }$, we have $q \leq q_{\max }$. Consider (15) for any $0<\mathrm{s} \leq \mathrm{s}_{\min }$. By contradicting hypothesis, $\hat{q}(\mathrm{~s}) \leq q_{\max }$. The above yields a Diophantine approximation of $\theta$ but not the optimal one as $\mathbf{S} \neq 0$. Now define

$$
(\tilde{p}, \tilde{q})=\arg \min _{(p, q) \in \mathbb{Z}^{\bar{N}+1}}\|p-\theta q\|_{X}
$$

along with

$$
\delta(\mathbf{s})=\|\hat{p}(\mathbf{s})-\theta \hat{q}(\mathbf{s})\|_{X}^{2}-\|\tilde{p}-\theta \tilde{q}\|_{X}^{2} .
$$

Observe that there exists a lower bound $\delta_{\min }$ such that $\delta(\mathrm{s}) \geq \delta_{\min }>0$ as $\| \hat{p}(\mathrm{~s})-$ $\theta \hat{q}(\mathrm{~s}) \|_{X}$ cannot reach its minimum since $\hat{q}(\mathrm{~s}) \leq q_{\mathrm{max}}$. Now, consider the original problem (15) with $\mathrm{S}<\min \left\{\frac{\sqrt{\delta_{\min }}}{\sqrt{2} \tilde{q}}, \mathrm{~S}_{\min }\right\}$. With this choice, we have

$$
(\mathrm{s} \tilde{q})^{2}<\frac{\delta_{\min }}{2}<\frac{\delta(\mathrm{s})}{2}<\frac{\delta(\mathrm{s})}{2}+(\mathrm{s} \hat{q}(\mathrm{~s}))^{2} .
$$

Then we have

$$
\begin{aligned}
\left\|\left(\begin{array}{c}
\tilde{p}-\theta \tilde{q} b \\
\mathrm{~s} \tilde{q}
\end{array}\right)\right\|_{\operatorname{diag}(X, 1)}^{2} & =\|\tilde{p}-\theta \tilde{q}\|_{X}^{2}+(\mathrm{s} \tilde{q})^{2} \\
& \leq\|\hat{p}(\mathrm{~s})-\theta \hat{q}(\mathrm{~s})\|_{X}^{2}-\delta(\mathrm{s})+(\mathrm{s} \hat{q}(\mathrm{~s}))^{2}+\frac{\delta(\mathrm{s})}{2} \\
& =\|\hat{p}(\mathrm{~s})-\theta \hat{q}(\mathrm{~s})\|_{X}^{2}+(\mathrm{s} \hat{q}(\mathrm{~s}))^{2}-\frac{\delta(\mathrm{s})}{2} \\
& =\left\|\left(\begin{array}{c}
\hat{p}(\mathrm{~s})-\theta \hat{q}(\mathrm{~s}) \\
\mathrm{s} \hat{q}(\mathrm{~s})
\end{array}\right)\right\|_{X \oplus 1}^{2}-\frac{\delta(\mathrm{s})}{2} \\
& <\left\|\left(\begin{array}{c}
\hat{p}(\mathrm{~s})-\theta \hat{q}(\mathrm{~s}) \\
\mathrm{s} \hat{q}(\mathrm{~s})
\end{array}\right)\right\|_{X \oplus 1}^{2}-\frac{\delta_{\min }}{2} .
\end{aligned}
$$

The above is clearly a contradiction to the optimality of $(\hat{p}(\mathrm{~s}), \hat{q}(\mathrm{~s}))$.

Note that the result appears trivial from Eq. (14) except that the behavior of the last component of the first vector of the reduced basis has not yet been explored in the weighted case.

Comparison between the weighted LLL-algorithm, $X(\theta q-p)$, and the one of Sect. 3.3, $Y^{-1}(\theta q-Y \bar{p})$, indicates that a good choice of the weighting might be $X=Y^{-1}$. This is only a guiding idea, as $X=Y^{-1}$ would mean that $Y \bar{p}=p$, that is, $Y \times \operatorname{Dirichlet}$ numerator $\left(Y^{-1} \theta\right)=$ Dirichlet numerator $(\theta)$, which does not hold exactly. 
For practical computation of the time steps $\tau=q$, we must find numerators $p_{k \ell}$ that fulfill the odd/even constraints using the LLL-algorithm. The nonuniform variant introduced above makes it simpler to find suitable parameters $X$ and $\mathrm{s}$, but a search is still required. To automate the search, we use a standard genetic algorithm to find weight vectors $X$ with a user-defined $s$ that minimize the number of parity constraint violations of the $p_{k \ell}$. This works well in most cases, requiring only a few iterations (typically up to 5) for reasonably sized populations (about 200). We suggest that the standard crossover and mutation operators could be adjusted to improve the performance of the search. In particular, increasing the likelihood of changing the $X$ values corresponding to denominators $p_{k \ell}$ that violate a constraint and increasing the likelihood of retaining $X$ values for which the corresponding $p_{k \ell}$ do not violate the constraints may improve performance.

Example 8 (Weighted LLL-Algorithm.) Consider a ring with $N=7$ spins and $p_{\max }(1,3)$. There are four eigenspaces with projectors $\Pi_{k}$, and three rationally independent transition frequencies $\omega_{k, k+1}=(-0.7530,-1.6920,-1.3569) / \pi$. Noting that $s_{k}=\operatorname{Sgn}\left\langle 3\left|\Pi_{k}\right| 1\right\rangle$ for $k=0, \ldots, 3$ yielding $\mathbf{s}=\left(s_{0}, s_{1}, s_{2}, s_{3}\right)=(1,-1,-1,1)$, we choose $\omega_{12}$ as reference frequency and set

$$
\boldsymbol{\theta}=2 \omega_{12}^{-1}\left(\omega_{01}, \omega_{23}\right)^{T}=(0.8901,1.6039)^{T}
$$

with corresponding constraints $\mathbf{s}^{\prime}=(1,1)$, which means that the numerators $p_{k}$ in the simultaneous Diophantine approximation of $\boldsymbol{\theta}$ must both be odd.

Applying the classical LLL-algorithm to solve the simultaneous Diophantine approximation for $\theta$ yields rational approximations of very high accuracy, as shown in Fig. 1a. However, most of the resulting approximations $p_{k} / q$ do not satisfy the parity constraints. Using the weighted LLL-algorithm and varying the diagonal scaling vector $X$ enable us to find solutions of arbitrary accuracy, as shown in Fig. 1b, all of which satisfy the parity constraints for the numerators $p_{k}$.
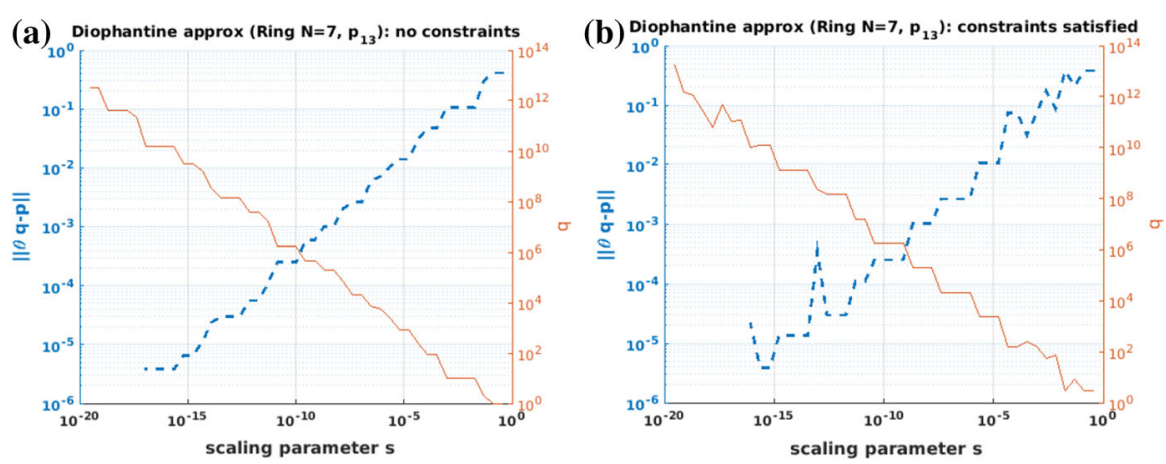

Fig. 1 Behavior of LLL-algorithm applied to the simultaneous Diophantine approximation to determine attainability of $p_{\max }(1,3)$ in a $N=7$ ring. The left vertical axis in both plots corresponds to the error of the approximation (thick broken line), while the right vertical axis corresponds to the transfer time (thin solid line). a Unweighted LLL-algorithm b Weighted LLL-algorithm 
For the approximation $p=(170921,307989)$ and $q=192028$, we obtain the transfer time $t_{f}=2 q / \omega_{12}=7.1308 \times 10^{5}$ (in units of $J^{-1}$ ) and corresponding transfer fidelity

$$
p_{t_{f}}(1,3)=\left|\sum_{k=0}^{4} \mathrm{e}^{-l \lambda_{k} t_{f}}\left\langle 3\left|\Pi_{k}\right| 1\right\rangle\right|^{2} \approx 0.4122,
$$

which is within $1-p_{t_{f}}(1,3) / p_{\max }(1,3)=2.41 \times 10^{-6}$ of the maximum transfer fidelity $p_{\max }(1,3)$.

The previous example illustrates how we can use the weighted LLL-algorithm to find optimal transfer times that yield very high transfer fidelities, and how we can control the margins of error and ensure the parity constraints are satisfied by adjusting the scaling parameter and diagonal weights in the algorithm.

\subsection{Estimate of time to attain maximum probability}

Our objective is to find an upper bound on the amount of time $t$ it takes to achieve $p_{t}(i, j) \geq p_{\max }(i, j)-\epsilon_{\mathrm{prob}}$, i.e., $p_{\max }(i, j)-p_{t}(i, j) \leq \epsilon_{\text {prob. }}$. The approach is to translate the specification on the probability $\epsilon_{\text {prob }}$ to a specification on the infinity norm of the simultaneous Diophantine approximation $\left\|\epsilon_{\mathrm{Da}}\right\|_{\infty}$, where $\epsilon_{\mathrm{Da}}=\theta q-p$.

Proceeding from (4), recalling that $\operatorname{Sgn}\left(\left\langle i\left|\Pi_{k}\right| j\right\rangle\right)=: s_{k}=\mathrm{e}^{-\imath \pi\left(s_{k}-1\right) / 2-2 \pi \imath n_{k}}$, where $n_{k}$ is some integer, we obtain

$$
\begin{aligned}
\sqrt{p_{\max }(i, j)} & =\left|\sum_{k=0}^{\widetilde{N}-1}\left\langle i\left|P i_{k}\right| j\right\rangle s_{k}\right| \\
& =\left|\sum_{k \in K^{\prime}}\left\langle i\left|\Pi_{k}\right| j\right\rangle \mathrm{e}^{-l \frac{\pi}{2}\left(s_{k}-1\right)-2 \pi \imath n_{k}}\right| \\
& =\left|\sum_{k \in K^{\prime}}\left\langle i\left|\Pi_{k}\right| j\right\rangle^{-l \frac{\pi}{2}\left(s_{k}-1\right)-2 \pi \imath n_{k}} \mathrm{e}^{l \frac{\pi}{2}\left(s_{\ell}-1\right)+2 \pi \imath n_{\ell}}\right| \\
& =\left|\sum_{k \in K^{\prime}}\left\langle i\left|\Pi_{k}\right| j\right\rangle \mathrm{e}^{-l \frac{\pi}{2}\left(s_{k}-s_{\ell}\right)-2 \pi \imath\left(n_{k}-n_{\ell}\right)}\right|
\end{aligned}
$$

In the second equation, the sum over $k$ has been replaced by a sum over $k \in K^{\prime}$ as states with $\left\langle i\left|\Pi_{k}\right| j\right\rangle=0$ do not contribute to the sum. The third equality stems from the fact that for fixed $\ell, \mathrm{e}^{l \frac{\pi}{2}\left(s_{\ell}-1\right)+2 \pi i n_{\ell}}$ is a global phase factor that is absorbed by the absolute value.

Next, we introduce the attainability condition (8), which is only approximately satisfied using the simultaneous Diophantine approximation. The idea is to expose the gap between the left-hand side and the right-hand side of (8) when $t$ is constrained to emerge from the Diophantine approximation: 


$$
\begin{aligned}
& \sqrt{p_{\max }(i, j)} \\
= & \left|\sum_{k \in K^{\prime}}\left(\left\langle i\left|\Pi_{k}\right| j\right\rangle \mathrm{e}^{-l\left(\lambda_{k}-\lambda_{\ell}\right) t}+\left\langle i\left|\Pi_{k}\right| j\right\rangle\left(\mathrm{e}^{-l \frac{\pi}{2}\left(s_{k}-s_{\ell}\right)-2 \pi l\left(n_{k}-n_{\ell}\right)}-\mathrm{e}^{-l\left(\lambda_{k}-\lambda_{\ell}\right) t}\right)\right)\right| \\
\leq & \left|\sum_{k \in K^{\prime}}\left\langle i\left|\Pi_{k}\right| j\right\rangle \mathrm{e}^{-l\left(\lambda_{k}-\lambda_{\ell}\right) t}\right|+\left|\sum_{k \in K^{\prime}}\left\langle i\left|\Pi_{k}\right| j\right\rangle\left(\mathrm{e}^{-l \frac{\pi}{2}\left(s_{k}-s_{\ell}\right)-2 \pi l\left(n_{k}-n_{\ell}\right)}-\mathrm{e}^{-l\left(\lambda_{k}-\lambda_{\ell}\right) t}\right)\right| \\
= & \left|\sum_{k \in K^{\prime}}\left\langle i\left|\Pi_{k}\right| j\right\rangle \mathrm{e}^{-l \lambda_{k} t}\right|+\left|\sum_{k \in K^{\prime}}\left\langle i\left|\Pi_{k}\right| j\right\rangle\left(\mathrm{e}^{-l \frac{\pi}{2}\left(s_{k}-s_{\ell}\right)-2 \pi l\left(n_{k}-n_{\ell}\right)}-\mathrm{e}^{-l\left(\lambda_{k}-\lambda_{\ell}\right) t}\right)\right| \\
\leq & \sqrt{p_{t}(i, j)}+\sum_{k \in K^{\prime}}\left|\mathrm{e}^{-l \frac{\pi}{2}\left(s_{k}-s_{\ell}\right)-2 \pi l\left(n_{k}-n_{\ell}\right)}-\mathrm{e}^{-l\left(\lambda_{k}-\lambda_{\ell}\right) t}\right| .
\end{aligned}
$$

It follows that $\sqrt{p_{\max }(i, j)}-\sqrt{p_{t}(i, j)} \leq \sum_{k \in K^{\prime}}\left|\mathrm{e}^{-l \frac{\pi}{2}\left(s_{k}-s_{\ell}\right)}-\mathrm{e}^{-l\left(\lambda_{k}-\lambda_{\ell}\right) t}\right|$. The trivial identity

$$
p_{\max }-p_{t}=\left(\sqrt{p_{\max }}-\sqrt{p_{t}}\right)\left(\sqrt{p_{\max }}+\sqrt{p_{t}}\right)
$$

then shows that to secure $p_{\max }(i, j)-p_{t}(i, j) \leq \epsilon_{\mathrm{prob}}$, it suffices to require

$$
\sum_{k \in K^{\prime}}\left|\mathrm{e}^{-l \frac{\pi}{2}\left(s_{k}-s_{\ell}\right)}-\mathrm{e}^{-l\left(\lambda_{k}-\lambda_{\ell}\right) t}\right| \leq \frac{\epsilon_{\mathrm{prob}}}{2},
$$

where it is observed that $\ell \in K^{\prime}$ is arbitrary.

The last step is to relate the left-hand side of (18) to the simultaneous Diophantine approximation error. Define

$$
\epsilon_{\mathrm{Da}}(k, \ell):=\left|\theta_{k \ell} q-p_{k \ell}\right|, \quad\left\|\epsilon_{\mathrm{Da}}\right\|_{\infty}=\max _{(k, \ell) \in \mathcal{S}} \epsilon_{\mathrm{Da}}(k, \ell),
$$

where $\mathcal{S}$ is the subset of linearly independent attainability equations chosen. By definition, any constraint $c_{k \ell}:=\omega_{k \ell} t-\frac{1}{2}\left(s_{k}-s_{\ell}\right)=0 \bmod 2$ with $\omega_{k \ell}=\left(\lambda_{k}-\lambda_{\ell}\right) / \pi$ can be written as a linear combination of constraints with $\left(k^{\prime}, \ell^{\prime}\right) \in \mathcal{S}, c_{k \ell}=$ $\sum_{\left(k^{\prime}, \ell^{\prime}\right) \in \mathcal{S}} b_{k^{\prime} \ell^{\prime}} c_{k^{\prime} \ell^{\prime}}$, with coefficients $b_{k^{\prime} \ell^{\prime}} \in\{0, \pm 1\}$. Furthermore, given $\omega_{m n} \in \mathcal{S}$ with $s_{m}=s_{n}$ and setting $t=2 \tau / \omega_{m n}$ with $\tau \in \mathbb{N}$ and $\theta_{k \ell}=2 \omega_{k \ell} / \omega_{m n}$, we can write the constraints as $c_{k \ell}=\theta_{k \ell} \tau-\frac{1}{2}\left(s_{k}-s_{\ell}\right)$ for $(k, \ell) \in \mathcal{S}_{0}$. Given a Diophantine approximation that satisfies the parity constraints, we have $c_{k \ell}=\epsilon_{\mathrm{Da}}(k, \ell) \bmod 2$ for $(k, \ell) \in \mathcal{S}$, and $c_{k \ell} \leq \bar{N}\left\|\epsilon_{\mathrm{Da}}\right\|_{\infty}$ for $(k, \ell) \notin \mathcal{S}$, where $\bar{N}=|\mathcal{S}|-1$ is the number of independent constraints reduced by 1 . Thus, we have

$$
\begin{aligned}
\sum_{k \in K^{\prime}}\left|\mathrm{e}^{-l \frac{\pi}{2}\left(s_{k}-s_{\ell}\right)}-\mathrm{e}^{-\imath \pi \omega_{k \ell} t}\right| & =\sum_{k \in K^{\prime}}\left|1-\mathrm{e}^{-\imath \pi c_{k \ell}}\right| \\
& \leq\left|K^{\prime}\right| \max _{k \in K^{\prime}}\left|1-\mathrm{e}^{-\imath \pi c_{k \ell}}\right| \\
& \leq 2\left|K^{\prime}\right|\left|\sin \left(\frac{\pi}{2} \bar{N}\left\|\epsilon_{\text {Da }}\right\|_{\infty}\right)\right|
\end{aligned}
$$


From the above string of inequalities, it follows that for the attainability accuracy $\epsilon_{\text {prob }}$ to be reached, it is sufficient to take

$$
2\left|K^{\prime}\right|\left|\sin \left(\frac{\pi}{2} \bar{N}\left\|\epsilon_{\mathrm{Da}}(q)\right\|_{\infty}\right)\right|<\frac{\epsilon_{\mathrm{prob}}}{2} .
$$

Although conservatively derived, the above formula is consistent with the tightly derived simulation results in Fig. 7 (right). We now summarize the situation we have reached:

Theorem 3 For homogeneous rings, the ITF specification $p_{t}(i, j) \geq p_{\max }(i, j)-$ $\epsilon_{\text {prob }}$ is achieved at time $t=2 q / \omega_{m n}$ (in $1 / J$ units) if $q$ is chosen so that simultaneous Diophantine approximation error $\epsilon_{\mathrm{Da}}(q):=\mathbf{p}-\boldsymbol{\theta} q$ has its infinity norm satisfying (20) and $\omega_{m n}$ is the reference transition with respect to which $\boldsymbol{\theta}=\left(\theta_{k \ell}\right)$ was defined in (10).

There are many simultaneous Diophantine approximation schemes. If we retain the Dirichlet-good one with even/odd constraints on the numerators, under the assumption that the algorithm of Sect. 3.3 converges, the error bound is $\left\|\epsilon_{\mathrm{Da}}\right\|_{\infty} \leq 2 / q^{1 / \bar{N}}$, and we obtain the further sufficient condition

$$
2\left|K^{\prime}\right|\left|\sin \left(\frac{\pi \bar{N}}{q^{1 / \bar{N}}}\right)\right|<\frac{\epsilon_{\text {prob }}}{2} .
$$

A minimum $q$ that guarantees $\epsilon_{\text {prob }}$ is easily extracted from the above inequality

$$
q \geq\left(\frac{\pi \bar{N}}{\sin ^{-1}\left(\frac{\epsilon_{\text {prob }}}{4\left|K^{\prime}\right|}\right)}\right)^{\bar{N}} \approx\left(\frac{4 \pi \bar{N}\left|K^{\prime}\right|}{\epsilon_{\text {prob }}}\right)^{\bar{N}},
$$

where the latter approximation uses $\sin (x) \approx x$ and is valid if $x=\frac{1}{4\left|K^{\prime}\right|} \epsilon_{\text {prob }} \ll 1$.

As an example will soon show, contrasting the above with numerical simulations of Eq. (4) reveals that the bound $O\left(\bar{N}^{\bar{N}}\right)$ is very conservative, mainly because the continuous-time dynamics on the torus was converted to a discrete-time dynamics. The conservativeness is somewhat mitigated by the dimension reduction achieved by the elimination of dark states and symmetries that reduce the number of relevant eigenspaces. For example, for homogeneous rings $\bar{N} \approx \frac{1}{2} N$ rather than $N$. Further improvement of the scaling behavior could be achieved by utilizing tighter simultaneous Diophantine approximations [13, Th.2], [23], but at the expense of significantly complicating the notation. The reward for the conservativeness of this bound is that it is quite general for rings with uniform coupling and their ITF attainable by the algorithm of Sect. 3.3, as it depends on neither the eigenvalues nor the odd/even pattern. Furthermore, it becomes very general for any network subject to the mild modification of replacing $\bar{N}$ by $N$ and $\left|K^{\prime}\right|$ by $N$.

Example 9 (Transfer Times-simulation results vs bounds.) For a ring with $N=5$, we have $\widetilde{N}=3$ independent eigenspaces with two rationally independent transition 

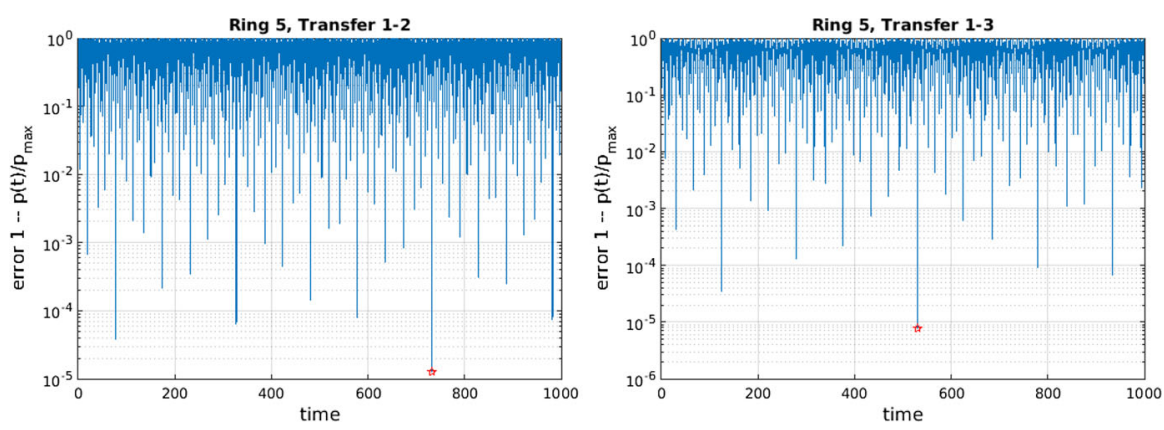

Fig. 2 Simulations of transfer probabilities from $1 \rightarrow 2$ and $1 \rightarrow 3$ for a ring of size $N=5$
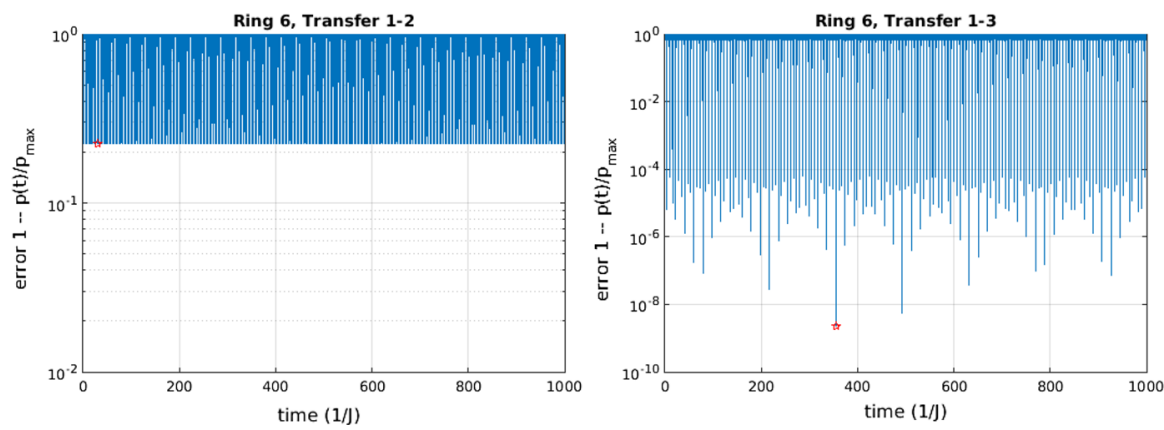

Fig. 3 Simulations of transfer probabilities from $1 \rightarrow 2$ and $1 \rightarrow 3$ for a ring of size $N=6$

frequencies and there are no dark subspaces. Hence, $\left|K^{\prime}\right|=3$ and we have $\bar{N}=1$ independent $\theta$. In this case, our conservative bound implies that we can get within $\epsilon_{\text {prob }}$ of the maximum transition probability in time $12 \pi / \epsilon_{\text {prob }}$.

In practice, simulations suggest that we can achieve very high fidelities in much shorter times. Figure 2 shows that we can achieve $>99.99 \%$ of the maximum transfer fidelity for any two nodes with distance 1 in time $t=77.28$, and transfer between two nodes with distance 2 in time $t=125$ (in units of $1 / J$ ). Notice that the maximum distance between any two nodes in a homogeneous ring of size $N=5$ is 2 , and hence any transfer can be achieved to within $0.01 \%$ of the maximum possible in time $t \leq 125$.

As observed before, for rings of size $N=6$, the primary transition frequencies are not rationally independent, implying that we do not have simultaneous attainability. Indeed, Fig. 3 (left) shows that the bound $p_{\max }(1,2)$ is not attainable. Lack of simultaneous attainability does not imply that all bounds are not attainable. Indeed Fig. 3 (right) suggests near-perfect transfer between nodes of distance $n=2$.

We can use simulations combined with the LLL-algorithm to estimate the minimum times required to achieve various transfers with a certain maximum error probability. The results for rings of size $N=5$ and $N=7$, which satisfy the rational independence conditions for simultaneous attainability, as shown in Fig. 4, suggest a power-law scaling. 

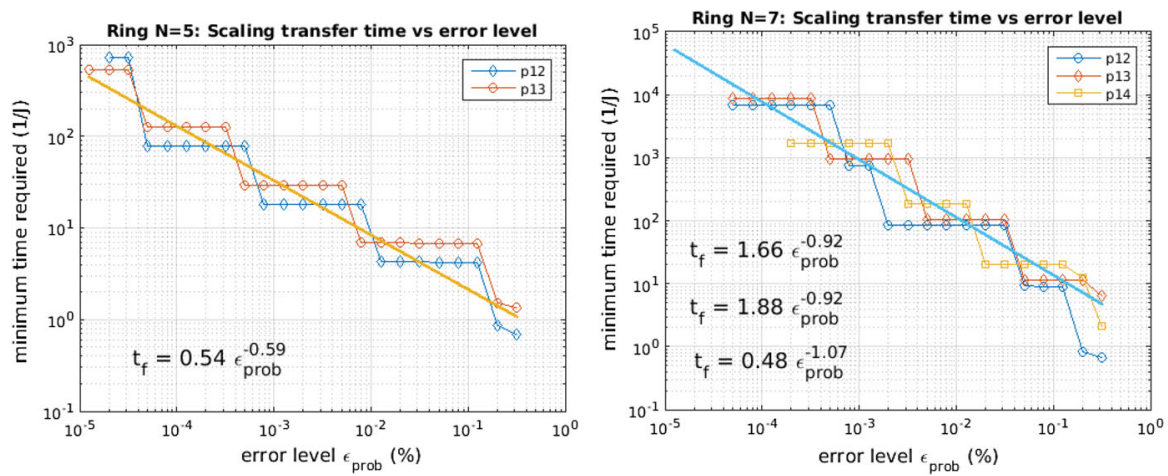

Fig. 4 Transfer times estimated from simulations versus the error probability for ring of size 5 (left) and 7 (right)
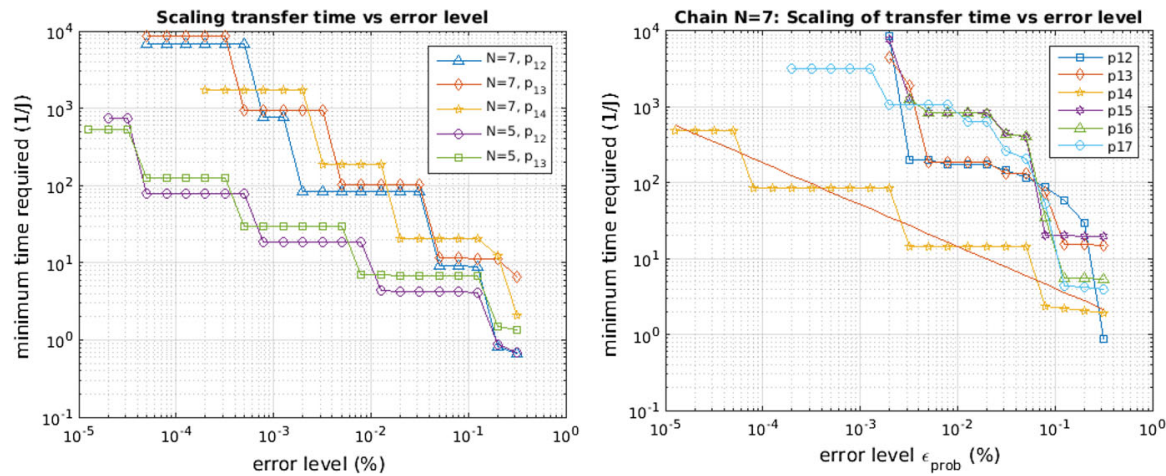

Fig. 5 Comparison of scaling of transfer times with error probability for rings (left) and scaling for chains of size $N=7$ (right)

Finally, comparing the scaling of the transfer times for rings of different size in Fig. 5(left) suggests that we have similar scalings for both $N=5$ and $N=7$ although the constant is larger for $N=7$. The scaling behavior for various transfers for a chain of size $N=7$ in Fig. 5(right) is similar but more complicated, and the transfer times required to get close to the upper bounds appear to be significantly longer.

\subsection{Transfer time versus decoherence time}

In general there is a trade-off between the error $\epsilon_{\text {prob }}$ and the transfer time $t_{f}$ required to achieve $p_{t_{f}}(i, j)=1-\epsilon_{\mathrm{prob}}$. For actual physical realizations of quantum networks, decoherence is generally a limiting factor. In this case, the relationship between the error probability and the expected transfer time can be useful in estimating what error probabilities can be achieved based on the coherence time of the network $t_{\mathrm{coh}}$.

For instance, in example 9, we showed that for a ring of size $N=5$, we would achieve $99 \%$ of the maximum transfer probability between any two nodes in time $T \leq 125$ in units of the inverse coupling rate $J^{-1}$, and we could therefore expect 
to closely approximate these transfer fidelities, provided the coherence time of the system is $\gg 100 \mathrm{~J}^{-1}$.

More generally, Figs. 4-5 suggest that we have the power law $t_{f}=c \epsilon_{\text {prob }}^{-\alpha}$ (in $1 / J$ units), at least for certain types of networks such as rings. In this case for the algorithm to work, it is necessary that

$$
c \epsilon_{\text {prob }}^{-\alpha} \ll t_{\text {coh }} .
$$

This means that realistically, the error probabilities $\epsilon_{\text {prob }}$ attainable are limited and we can expect

$$
\epsilon_{\text {prob }} \gg\left(c / t_{\mathrm{coh}}\right)^{1 / \alpha},
$$

and the algorithm of Sect. 3.3 could be used to construct a simultaneous Diophantine approximation compatible with this requirement.

Combining Theorem 3 and Eq. (21) also yields an upper bound on the transfer times for which the effect of decoherence should definitively be negligible

$$
t_{f} \leq \frac{2}{\omega_{m n}}\left(\frac{\pi \bar{N}}{\sin ^{-1}\left(\frac{\left(c / t_{\mathrm{coh}}\right)^{1 / \alpha}}{4\left|K^{\prime}\right|}\right)}\right)^{\bar{N}}
$$

although we would like to stress here that this bound is excessively conservative due to the approximations made. Given a concrete physical realization of a quantum network with an specific decoherence model, this information could be used to derive tighter time-dependent bounds on the transfer fidelities and realistic transfer times.

\section{Information transfer (in-)fidelity metric and geometry}

In this section, we come back to an issue raised in Sect. 3-namely that the upper bound derived in Eq. (4) can be justified by the fact that it induces a metric on the set of vertices. Unlike the results in the previous sections, most of the results in this section apply specifically to rings, although numerical simulations suggest that similar results may hold for other homogeneous spin networks such as chains.

\subsection{Definition and motivation of ITF prametric}

To develop a geometric picture, we can view a spin network as a pre-metric or more precisely a prametric space ${ }^{2}$ endowed with the prametric that quantifies the information transfer infidelity $(I T I)$. To fix terminology, recall that given a graph $\mathcal{G}=(\mathcal{V}, \mathcal{E})$, or any set of points $\mathcal{V}$ for that matter, a prametric [33, p. 666], [32, p.23] is a function $d: \mathcal{V} \times \mathcal{V} \rightarrow \mathbb{R}_{\geq 0}$ such that (i) $d(i, j) \geq 0$ and (ii) $d(i, i)=0$.

To derive a suitable prametric on the vertex set $\mathcal{V}=\{|i\rangle: i=1, \ldots, N\}$ from the probability $p_{\max }$ data, we inspire ourselves from a similar situation in sensor networks [34], where $\mathcal{V}$ is the set of sensors and a Packet Reception Rate $\operatorname{PRR}(i, j)$

\footnotetext{
2 We prefer to avoid the terminology of pre-metric space since it is not quite accepted; prametric on the other hand is the terminology introduced by Arkhangel'skii and Pontryagin [32].
} 
is defined as the probability of successful transmission of the packets from sensor $i$ to sensor $j$. After symmetrization of the packet reception rate, a prametric (in fact, a semi-metric [35-37]) can be defined as $d(i, j)=-\log \operatorname{PRR}(i, j)$. Should there be a violation of the triangle inequality, say, $d(i, j)>d(i, k)+d(k, j)$, then the distance between $i$ and $j$ is redefined as $d(i, k)+d(k, j)$. The importance of the metric is that it provides a notion of network curvature, which has a dramatic impact on the traffic flow [38,39] in a paradigm that extends to quantum chains [14]. Following sensor network intuition[34], we define

$$
d(i, j)=-\log p_{\max }(i, j) .
$$

Obviously, $d(i, j) \geq 0$ and, as will be shown in Theorem $4, d(i, i)=0$.

We could define the time-stamped prametric by $d_{t}(i, j)=-\log p_{t}(|i\rangle,|j\rangle)$ except that in general $d_{t}(i, i) \neq 0$. To remedy this situation, we could define $d(i, j)=$ $\inf _{t \geq 0} d_{t}(i, j)=-\log \sup _{t \geq 0} p_{t}(i, j)$. Since, by Cauchy-Schwarz, $p_{t}(i, i) \leq 1$ and $p_{t=0}(i, i)=1$, we have $\sup _{t \geq 0} p_{t}(i, i)=1$ and hence $d(i, i)=0$. This alternate prametric definition is equivalent to the earlier one when $p_{\max }$ is attainable, but it reveals that this prametric makes the network of finite diameter $\left(\sup _{i, j} d(i, j)<\infty\right)$ as $N \rightarrow \infty$ as Theorem 4 will show. This has the unfortunate consequence of preventing a genuine large-scale analysis. As Sect. 5 will show, a bias rectifies this problem (see also [14]).

Generally, this information transfer infidelity prametric is not a proper distance satisfying the triangle inequality, but for certain networks such as rings with uniform coupling this prametric will be shown to define a proper distance.

This quantum mechanical (pra)metric is quite different from the usual Euclidean distance $d_{\mathbb{E}}$ of the spins in the spintronic device. In particular, two spins that are physically close in the medium may be far quantum mechanically, and conversely. If two spins are quantum mechanically far, control is necessary to enable transmissions that are too weak or forbidden by the natural quantum mechanical couplings. This control of information can be viewed as the problem of controlling the quantum mechanical geometry of the network.

\subsection{ITF distance geometry of homogeneous spin rings}

It could be argued that a prametric is sufficient if we are solely interested in assessing the difficulty of communication or fidelity of information transfer between nodes in a network. However, a proper metric allows us to investigate other geometric properties such as the curvature of the network with regard to the ITF.

A prametric $d: \mathcal{V} \times \mathcal{V} \rightarrow \mathbb{R}_{\geq 0}$ is a pseudo-metric if in addition to (i) $d(i, j) \geq 0$, $d(i, i)=0$, it satisfies (ii) $d(i, j)=d(j, i)$ and (iii) the triangle inequality $(d(i, j) \leq$ $d(i, k)+d(k, j))$ holds. A metric or distance is a pseudo-metric that has (iv) the separation property: $d(i, j)=0$ if and only if $i=j$.

Theorem 4 For a quantum ring $\left(\mathcal{V}_{N}, \mathcal{E}_{N}\right)$ of $N$ uniformly distributed spins with $X X$ or Heisenberg couplings, $d_{N}(i, j):=-\log p_{\max }(i, j)$ has the following properties:

1. For $N$ odd, $\left(\mathcal{V}_{N}, d_{N}\right)$ is a metric space. 
2. For $N$ even, $\left(\mathcal{V}_{N}, d_{N}\right)$ is a pseudo-metric space that becomes metric after antipodal point identification.

3. If $N=p$ or $N=2 p$, where $p$ is a prime number, then the distances on the space of equivalence classes of spins are uniform, i.e., $d_{N}(i, j)=c_{N}$ for $i \neq j$. Otherwise, the distances are nonuniform.

4. In all cases $\lim _{N \rightarrow \infty} d_{N}(i, j)=2 \log \frac{\pi}{2}, i \neq j \bmod \left(\frac{1}{2} N\right)$.

Proof To show that $\left(\mathcal{V}_{N}, d_{N}\right)$ is a pseudo-metric space, we need to verify that (i) $d_{N}(i, i)=0$, (ii) $d_{N}(i, j)=d_{N}(j, i)$, and (iii) the triangle inequality holds. For a metric space, we must further have (iv) $d_{N}(i, j) \neq 0$ unless $i=j$.

(i) is clearly satisfied as the projectors onto the eigenspaces are a resolution of the identity, $\sum_{k} \Pi_{k}=I$, and thus for any unit vector $|i\rangle$, we have $\sum_{k=1}^{N}\left|\left\langle i\left|\Pi_{k}\right| i\right\rangle\right|=$ $\sum_{k=1}^{N} \| \Pi_{k}|i\rangle \|^{2}=1$. (ii) follows from $\left|\left\langle i\left|\Pi_{k}\right| j\right\rangle\right|=\left|\left\langle j\left|\Pi_{k}\right| i\right\rangle\right|$. The proof of the remaining properties relies on the circulant matrix property of the Hamiltonian $\bar{H}$ in the single excitation subspace $\overline{\mathcal{H}}$, as shown in Eq. (3) and Table 1.

Observe in Table 1 the double eigenvalues $\lambda_{k}=\lambda_{N-k}$, except for $k=0$ and $k=\frac{1}{2} N$ if $N$ even. From Table 1, each of these double eigenvalues has two general complex conjugate eigenvectors. These general eigenvectors need not be orthogonal, but observing that $\left\langle v_{k} \mid v_{\ell}\right\rangle=\delta_{k \ell}$ and $\left\langle v_{k} \mid v_{k}^{*}\right\rangle=0$, where $v_{k}^{*}$ denotes the complex conjugate, it follows that

$$
\begin{aligned}
\left|\bar{v}_{0}\right\rangle & =\left|v_{0}\right\rangle=\frac{1}{\sqrt{N}}(1,1, \ldots)^{T}, \\
\left|\bar{v}_{k}\right\rangle & =\left|v_{k}\right\rangle, \quad\left|v_{N-k}\right\rangle=\left|v_{k}^{*}\right\rangle, \quad k=1, \ldots N^{\prime}=\left\lfloor\frac{N-1}{2}\right\rfloor, \\
\left|\bar{v}_{N / 2}\right\rangle & =\left|v_{N / 2}\right\rangle=\frac{1}{\sqrt{N}}(1,-1, \ldots)^{T}, \quad \text { if } N \text { is even, }
\end{aligned}
$$

defines an orthonormal basis of $\overline{\mathcal{H}}$. Furthermore, in the basis in which $\bar{H}$ is circulant, we have $|i\rangle=e_{i}$, where $\left\{e_{i}: i=1, \ldots, N\right\}$ is the natural basis of $\mathbb{C}^{N}$.

$$
\begin{aligned}
\left|\left\langle i\left|\Pi_{0}\right| j\right\rangle\right| & =\left|\left\langle i \mid \bar{v}_{0}\right\rangle\left\langle\bar{v}_{0} \mid j\right\rangle\right|=\frac{1}{N}, \\
\left|\left\langle i\left|\Pi_{k}\right| j\right\rangle\right| & =\left|\left\langle i \mid \bar{v}_{k}\right\rangle\left\langle\bar{v}_{k} \mid j\right\rangle+\left\langle i \mid \bar{v}_{N-k}\right\rangle\left\langle\bar{v}_{N-k} \mid j\right\rangle\right| \\
& =\left|\rho_{N}^{k i}\left(\rho_{N}^{k j}\right)^{*}+\left(\rho_{N}^{k i}\right)^{*} \rho_{N}^{k j}\right| \frac{1}{N} \\
& =\left|\rho_{N}^{k(i-j)}+\rho_{N}^{-k(i-j)}\right| \frac{1}{N}=\frac{2}{N}\left|\cos \left(\frac{2 \pi k(i-j)}{N}\right)\right|, \\
\left|\left\langle i\left|\Pi_{N / 2}\right| j\right\rangle\right| & =\left|\left\langle i \mid \bar{v}_{N / 2}\right\rangle\left\langle\bar{v}_{N / 2} \mid j\right\rangle\right|=\frac{1}{N} .
\end{aligned}
$$

Summing over all eigenspaces $k=0, \ldots,\lfloor N / 2\rfloor$ gives

$$
\sqrt{p_{\max }(i, j)}= \begin{cases}\frac{1}{N}+\frac{2}{N} \sum_{k=1}^{N^{\prime}}\left|\cos \left(\frac{2 \pi k(i-j)}{N}\right)\right|, & N=2 N^{\prime}+1 \\ \frac{2}{N}+\frac{2}{N} \sum_{k=1}^{N^{\prime}}\left|\cos \left(\frac{2 \pi k(i-j)}{N}\right)\right|, & N=2 N^{\prime}+2 .\end{cases}
$$

For $N=2 N^{\prime}+1$, it is easy to see that $p_{\max }(i, j)=1$ if and only if $i=j$, hence (iv). For $N=2 N^{\prime}+2$, on the other hand, we also have $\left|\cos \left(\frac{2 \pi k N / 2}{N}\right)\right|=|\cos (\pi k)|=1$, 
and thus $d(i, j)=0$ for $i-j=\frac{1}{2} N$, i.e., the distance vanishes for antipodal points, and thus $d(i, j)$ is at most a pseudo-metric. However, noting that we can identify antipodal points $|j\rangle$ and $\left|j+N^{\prime}+1\right\rangle$, let $d$ be defined on the set of equivalence classes $[|j\rangle]$ for $j=1, \ldots, N^{\prime}+1$ instead. (The antipodal identification preserves the ring structure.) At this stage, $d$ is a semi-metric $[35,36,40]$, that is, it satisfies all axioms of a metric except the triangle inequality.

To prove the triangle inequality, we show that $\sqrt{p_{\max }(i, m)} \sqrt{p_{\max }(m, j)} \leq$ $\sqrt{p_{\max }(i, j)}$. The definition (4) of $p_{\max }$ rewritten in terms of the eigenvectors of $\bar{H}$ using (27)-(29) gives

$$
\begin{aligned}
& \sqrt{p_{\max }(i, m)}=\frac{1}{N} \sum_{k=0}^{N-1} \alpha_{k} \rho_{N}^{k(m-i)}, \\
& \sqrt{p_{\max }(m, j)}=\frac{1}{N} \sum_{k^{\prime}=0}^{N-1} \beta_{k^{\prime}} \rho_{N}^{k^{\prime}(j-m)},
\end{aligned}
$$

where $\alpha_{k}=s_{k}(i, m)=\operatorname{Sgn}\left(\rho_{N}^{k(m-i)}+\rho_{N}^{-k(m-i)}\right) \in\{ \pm 1,0\}$ is rewritten explicitly in terms of the eigenvectors rather than as in Sect. 3 and $\beta_{k^{\prime}}=s_{k^{\prime}}(m, j)$. Setting

$$
\gamma_{k}=\sum_{k^{\prime}=0}^{N-1} \alpha_{k} \beta_{k^{\prime}} \rho_{N}^{\left(k^{\prime}-k\right)(j-m)}
$$

we obtain

$$
\begin{aligned}
& \sqrt{p_{\max }(i, m)} \sqrt{p_{\max }(m, j)} \\
= & \frac{1}{N^{2}} \sum_{k, k^{\prime}=0}^{N-1} \alpha_{k} \beta_{k^{\prime}} \rho_{N}^{k(m-i)} \rho_{N}^{k^{\prime}(j-m)} \\
= & \frac{1}{N^{2}} \sum_{k, k^{\prime}=0}^{N-1} \alpha_{k} \beta_{k^{\prime}} \rho_{N}^{k(j-i)+\left(k^{\prime}-k\right)(j-m)} \\
= & \frac{1}{N^{2}} \sum_{k=0}^{N-1} \gamma_{k} \rho_{N}^{k(j-i)}=\left|\frac{1}{N^{2}} \sum_{k=0}^{N-1} \gamma_{k} \rho_{N}^{k(j-i)}\right| .
\end{aligned}
$$

The final equality follows because the LHS and thus the RHS are known to be real and positive. Furthermore, as $\rho_{N}$ is a root of unity, $\left|\rho_{N}\right|=1$, and recalling $\left|\alpha_{k}\right|=$ $\left|\beta_{k^{\prime}}\right|=1,0$,

$$
\begin{aligned}
\left|\gamma_{k}\right| & =\left|\rho_{N}^{k(m-j)} \sum_{k^{\prime}=0}^{N-1} \alpha_{k} \beta_{k^{\prime}} \rho_{N}^{k^{\prime}(j-m)}\right| \\
& \leq\left|\rho_{N}^{k(m-j)}\right| \cdot \sum_{k^{\prime}=0}^{N-1}\left|\alpha_{k} \beta_{k^{\prime}} \rho_{N}^{k^{\prime}(j-m)}\right| \leq N,
\end{aligned}
$$


where the last inequality allows for the presence of dark states. Again we have $\rho_{N}^{(N-k)(m-j)}=\rho_{N}^{-k(m-j)}$, and as the LHS above is known to be real, we know that we must have $\gamma_{k}=\gamma_{N-k}$. Hence, we can again collect exponential terms pairwise to obtain cosines, which gives for $N=2 N^{\prime}+1$ :

$$
\begin{aligned}
\left|\frac{1}{N^{2}} \sum_{k=0}^{N-1} \gamma_{k} \rho_{N}^{k(j-i)}\right| & =\left|\frac{\gamma_{0}}{N^{2}}+\frac{1}{N^{2}} \sum_{k=1}^{N^{\prime}} 2 \gamma_{k} \cos \left(\frac{2 \pi k(j-i)}{N}\right)\right| \\
& \leq \frac{\left|\gamma_{0}\right|}{N^{2}}+\frac{2}{N^{2}} \sum_{k=1}^{N^{\prime}}\left|\gamma_{k}\right|\left|\cos \left(\frac{2 \pi k(j-i)}{N}\right)\right| \\
& \leq \frac{1}{N}+\frac{2}{N} \sum_{k=1}^{N^{\prime}}\left|\cos \left(\frac{2 \pi k(j-i)}{N}\right)\right| \\
& =\sqrt{p_{\max }(i, j)} .
\end{aligned}
$$

For $N=2 N^{\prime}+2$, we simply replace $\gamma_{0}$ by $\gamma_{0}+\gamma_{N^{\prime}+1}$ above to obtain

$$
\begin{aligned}
\left|\frac{1}{N^{2}} \sum_{k=0}^{N-1} \gamma_{k} \rho_{N}^{k(j-i)}\right| & \leq \frac{2}{N}+\frac{2}{N} \sum_{k=1}^{N^{\prime}}\left|\cos \left(\frac{2 \pi k(j-i)}{N}\right)\right| \\
& =\sqrt{p_{\max }(i, j)} .
\end{aligned}
$$

This proves (iii) and hence parts (1) and (2) of the theorem.

To establish (3), we note that if $N=2 N^{\prime}+1$ is prime, then

$$
\sum_{k=1}^{N^{\prime}}\left|\cos \left(\frac{2 \pi k(i-j)}{N}\right)\right|=\sum_{k=1}^{N^{\prime}}\left|\cos \left(\frac{2 \pi k}{N}\right)\right| .
$$

If $N$ is not $p$ or $2 p$, then $N$ and $(i-j)$ will have factors (which can be canceled) in common for some $(i-j)$ but not for others and hence we will obtain different distances.

To establish (4), letting $N \rightarrow \infty$, it is easily seen that the dependency on $i, j$ is eliminated, provided $i \neq j \bmod \left(\frac{1}{2} N\right)$. Hence, taking the norm of the above and then $-\log (\cdot)$, it follows that at the infinite ring limit, the distance is uniform for $i \neq j+$ $\bmod \left(\frac{1}{2} N\right)$. Finally,

$$
\begin{aligned}
\lim _{N \rightarrow \infty} \sqrt{p_{\max }(i, j)} & =\lim _{N \rightarrow \infty} \frac{2}{N} \sum_{k=0}^{N / 2}|\cos ((i-j) 2 \pi k / N)| \\
& =\frac{2|i-j|}{\pi} \int_{0}^{\frac{\pi}{2|i-j|}} \cos (|i-j| x) d x \\
& =\frac{2|i-j|}{\pi|i-j|}[\sin (|i-j| x)]_{0}^{\frac{\pi}{2|i-j|}}=\frac{2}{\pi}
\end{aligned}
$$

shows that $\lim _{N \rightarrow \infty} d_{N}(i, j)=2 \log \frac{\pi}{2} \approx 2 \times 0.4516$ for $i \neq j \bmod (N / 2)$. 
Case 3 of Theorem 4 allows for a very specific geometrization of the quantum ring in terms of constant curvature spaces. Define the $n$-sphere of curvature $\kappa$ as $\mathbb{S}_{\kappa}^{n}:=\left\{x \in \mathbb{R}^{n+1}:\|x\|^{2}=1 / \kappa\right\}$. We have the following corollary:

Corollary 1 The metric space $\left(\mathcal{V}_{p}, d_{p}\right)$ of $p$ spins $(p \geq 3$ prime) arranged in a homogeneous ring with uniform ITI distance $d_{p}(i, j)=c_{p}, i \neq j$, is isometrically embeddable in $\mathbb{S}_{\kappa}^{p-1}$ iff

$$
\kappa \leq\left[\frac{1}{c_{p}} \cos ^{-1}\left(-\frac{1}{p-1}\right)\right]^{2} .
$$

Furthermore, it is irreducibly isometrically embeddable in $\mathbb{S}_{\kappa}^{p-2}$ for

$$
\kappa=\left[\frac{1}{c_{p}} \cos ^{-1}\left(-\frac{1}{p-1}\right)\right]^{2} .
$$

Notes: In the above, "irreducibly embeddable" means that the embedding cannot happen into a lower-dimensional constant curvature space. By convention, $\cos ^{-1}$ takes values in $[\pi / 2, \pi]$.

Proof This result is a corollary of [40, Th.63.1]. For the details, see [41, Appendix].

Note that this corollary deals with embeddability of the vertices only; however, edges can be mapped isometrically as arcs of great circles on either the sphere of curvature (31) or that of curvature (32). Also note that the symmetry of the simple $p=3$ case of the circle $\mathbb{S}^{1}$ circumscribed to a equilateral triangle is misleading, as in very high dimension $(p \rightarrow \infty)$, Eq. (32) yields $1 / \sqrt{\kappa}=: R \rightarrow \frac{c_{p}}{\pi / 2}$, that is, all vertices are mapped to the half-sphere of radius $R$.

Regarding $N=2 p$ in Case 3, we could first do the antipodal identification on the combinatorial ring $\left(\mathcal{V}_{2 p}, \mathcal{E}_{2 p}\right)$, leading to a $\left(\mathcal{V}_{p}, \mathcal{E}_{p}\right)$ ring, and then embed $\left(\mathcal{V}_{p}, \mathcal{E}_{p}\right)$ as in the preceding corollary.

Regarding Case 4 when $N$ is odd, define $\epsilon:=\max _{i \neq j}\left|d_{N}(i, j)-2 \log (\pi / 2)\right|$. Then the metric space $\left(\mathcal{V}_{N}, d_{N}\right)$ can be mapped isometrically on the sphere $\mathbb{S}_{\kappa}^{N-2}$ of radius $d_{\infty} / \cos ^{-1}\left(-(N-1)^{-1}\right)$ up to an additive distortion not exceeding $\epsilon$, that is, the embedding is quasi-isometric [42, 7.2.G]. The case of an even $N$ is dealt with as before using antipodal identification. The geometry of a genuinely infinite ring $(N=\infty$ rather than $N \rightarrow \infty)$ is completely different and is left to future work.

The $N$ even case can be dealt with in a different way. Rather than doing, first, a combinatorial antipodal identification $\left(i=j\right.$ if $\left.i-j=0 \bmod \left(\frac{1}{2} N\right)\right)$ and, then, mapping the quotient space $\mathcal{V}_{N} / \sim$ to the sphere, we could map the combinatorial antipodal points to geometrical antipodal points on the sphere $\mathbb{S}_{\kappa}^{N-2}$ with the understanding that geometrical antipodal points on the sphere are identified to yield the real projective space $\mathbb{R P}^{N-2}$. A slight generalization of (32) of Corollary 1 together with 4 of Theorem 4 yields an irreducible embedding of $\left(\mathcal{V}_{N}, d_{N}\right)$ into the sphere of curvature $\kappa=\left(\left(\cos ^{-1}\left(-\frac{1}{N-1}\right)\right) /\left(2 \log \frac{\pi}{2}\right)\right)^{2}$. On the other hand, $\mathbb{R P}^{N-2}$ is usually endowed with the standard curvature 1 metric of diameter $\pi / 2$. To sum up: 
Corollary 2 For $N$ even, there is an embedding $\mathcal{V}_{N} \hookrightarrow \mathbb{R P}^{N-2}$, which is quasiisometric for the scaled distance $d_{N} \cos ^{-1}\left(-\frac{1}{N-1}\right) /\left(4 \log \frac{\pi}{2}\right)$ on $\mathcal{V}_{N}$ and the curvature 1 distance on $\mathbb{P R}^{N-2}$. Furthermore, for $N \rightarrow \infty$ the distortion becomes vanishingly small.

\section{Control of information transfer fidelity}

To overcome intrinsic limitations on quantum state transfer or speed up transfer, one can either try to engineer spin chains or networks with nonuniform couplings [6,7], or introduce dynamic control to change the network topology [9-11].

Our analysis above shows that engineering the couplings is not strictly necessary. For an XX or Heisenberg-type chain with uniform nearest-neighbor couplings, for example, it can easily be verified that the information transfer fidelity between the end spins is unity, and attainability of the bounds means that we can achieve arbitrarily high state transfer fidelities between the end spins if we wait long enough. Engineering the couplings, however, can speed up certain state transfer tasks such as state transfer between the end spins at the expense of others.

A more flexible alternative to fixed engineered couplings is to apply control to change the network geometry and hence speed up state transfer as well as enable some transfers that either were forbidden or had poor ITF. One way this can be achieved is to apply static electromagnetic bias fields to change the energy-level splittings between the spin-up and spin-down states for different nodes in the graph, as suggested e.g., in [12]. To see how the application of such bias fields can alter the transfer fidelities and network geometry, consider a simple, concrete example of a single bias field $\zeta$ applied to node $\ell$ in a spin ring with uniform coupling. First, due to translation invariance, we can always relabel the nodes so that the biased node is node $N$. Then, assuming XX coupling, the Hamiltonian on the single excitation subspace becomes

$$
\bar{H}_{N}^{(\zeta)}=\left(\begin{array}{ccccccccc}
0 & 1 & \ldots & 0 & 0 & 0 & \ldots & 0 & 1 \\
1 & 0 & \ldots & 0 & 0 & 0 & \ldots & 0 & 0 \\
\vdots & \vdots & \ddots & \vdots & \vdots & \vdots & & \vdots & \vdots \\
0 & 0 & \ldots & 0 & 1 & 0 & \ldots & 0 & 0 \\
0 & 0 & \ldots & 1 & 0 & 1 & \ldots & 0 & 0 \\
0 & 0 & \ldots & 0 & 1 & 0 & \ldots & 0 & 0 \\
\vdots & \vdots & & \vdots & \vdots & \vdots & \ddots & \vdots & \vdots \\
0 & 0 & \ldots & 0 & 0 & 0 & \ldots & 0 & 1 \\
1 & 0 & \ldots & 0 & 0 & 0 & \ldots & 1 & \zeta
\end{array}\right),
$$

where it is observed that we have the decomposition

$$
\bar{H}_{N}^{(\zeta)}=C_{N}+\zeta E_{N, N}
$$

where $C_{N}$ is the $N \times N$ circulant matrix defined above and $E_{N, N}$ is a $N \times N$ matrix which is zero except for a 1 at position $(N, N)$. 
Physically, applying a large bias field to the $N$ th node in the ring results in a large detuning that effectively eliminates this node from the ring and breaks the ring open, leaving a chain of length $N-1$. Hence, in the limit $\zeta \rightarrow \infty$, we expect the transition fidelities for the first $N-1$ nodes to approach those for a chain of length $N-1$ while the transition fidelities between the first $N-1$ nodes and the final (biased) node approach 0 . We now reformulate this intuitively obvious result in precise mathematical language.

Lemma 2 The eigenvalues and eigenvectors of the $(N-1) \times(N-1)$ Toeplitz matrix $T_{N-1}$ made up of ones on the super diagonal and subdiagonal and zeros everywhere else are given by $\lambda_{k}=2 \cos \left(\frac{\pi k}{N}\right)$ and $\left|v_{k}\right\rangle_{i}=\sqrt{\frac{2}{N}} \sin \left(\frac{\pi k i}{N}\right) ; k=1, \ldots, N-1$, $i=1, \ldots, N-1$. Furthermore, for $k$ even, $\left|v_{k}\right\rangle_{1}+\left|v_{k}\right\rangle_{N-1}=0$.

Theorem 5 Let $p_{\text {chain }}^{N-1}$ be the maximum transfer fidelities for a spin chain of length $N-1$ with uniform coupling between the nearest neighbors. Let $p_{\text {ring }}^{N, \zeta}$ be the maximum transfer fidelities for a ring of size $N$ with bias $\zeta$ on the N th node. Then

$$
\lim _{\zeta \rightarrow \infty} p_{\text {ring }}^{N, \zeta}(i, j)= \begin{cases}p_{\text {chain }}^{N-1}(i, j), & \text { if } i, j<N ; \\ 0, & i=N, j \neq N \text { or } i \neq N, j=N ; \\ 1, & i, j=N .\end{cases}
$$

Proof Write the characteristic polynomial of $\bar{H}_{N}^{\zeta}$ as $\operatorname{det}\left(\left(\lambda I_{N}-C_{N}\right)-\zeta E_{N, N}\right)$ and recall that the determinant of the sum of two matrices equals the sums of the determinants of all matrices made up with some columns of one matrix and the complementary columns of the other matrix. Applying the latter to the characteristic polynomial of $\bar{H}_{N}^{(\zeta)}$ yields

$$
\operatorname{det}\left(\lambda I_{N}-\bar{H}^{(\zeta)}\right)=\operatorname{det}\left(\lambda I_{N}-C_{N}\right)-\zeta \operatorname{det}\left(\lambda I_{N-1}-T_{N-1}\right)
$$

where $T_{N-1}$ is the Toeplitz matrix defined in the lemma. From classical root-locus techniques, it follows that as $\zeta \rightarrow \infty$, exactly one eigenvalue $\lambda_{N}(\zeta)$ goes to $\infty$, while the remaining ones $\lambda_{1}(\zeta), \ldots, \lambda_{N-1}(\zeta)$ converge to the roots of $\operatorname{det}\left(\lambda I_{N-1}-T_{N-1}\right)=$ 0 .

Next, we look at the eigenvectors and rewrite the eigenvector equation as

$$
\left(\begin{array}{c|c} 
& 1 \\
T_{N-1} & 0_{N-3} \\
& 1 \\
\hline 10_{N-3} & \zeta
\end{array}\right)\left(\begin{array}{c}
\left|v_{k}(\zeta)\right\rangle_{1} \\
\vdots \\
\frac{\left|v_{k}(\zeta)\right\rangle_{N-1}}{\left|v_{k}(\zeta)\right\rangle_{N}}
\end{array}\right)=\lambda_{k}(\zeta)\left(\begin{array}{c}
\left|v_{k}(\zeta)\right\rangle_{1} \\
\vdots \\
\frac{\left|v_{k}(\zeta)\right\rangle_{N-1}}{\left|v_{k}(\zeta)\right\rangle_{N}}
\end{array}\right)
$$

Consider first the first $k \neq N$ equations. Since $\lim _{\zeta \rightarrow \infty} \lambda_{k}(\zeta)$ exists and is finite, it follows from the bottom eigenequation that $\zeta\left|v_{k}(\zeta)\right\rangle_{N}$ remains bounded as $\zeta \rightarrow \infty$. Therefore, $\lim _{\zeta \rightarrow \infty}\left|v_{k}\right\rangle_{N}=0$. Since $\lambda_{k}(\infty)$ is a unique eigenvalue of $T_{N-1}$, it follows that $\lim _{\zeta \rightarrow \infty}\left|v_{k}(\zeta)\right\rangle_{1: N-1}$ is the corresponding eigenvector of $T_{N-1}$. It remains to 
show that with this $\left|v_{k}\right\rangle_{1: N-1}$ the bottom eigenequation can be made to hold. This is easily achieved by defining

$$
\lim _{\zeta \rightarrow \infty} \zeta\left|v_{k}(\zeta)\right\rangle_{N}=-\lim _{\zeta \rightarrow \infty}\left(\left|v_{k}(\zeta)\right\rangle_{1}+\left|v_{k}(\zeta)\right\rangle_{N-1}\right)
$$

By the lemma, for $k$ even, we have $\lim _{\zeta \rightarrow \infty} \zeta\left|v_{k}(\zeta)\right\rangle_{N}=0$, and therefore the $k<N$ eigenequation holds with $\left|v_{k}(\zeta)\right\rangle_{N}$ going to zero faster than $1 / \zeta$. For $k$ odd, $\left|v_{k}(\zeta)\right\rangle_{N}$ goes to zero as $c / \zeta$, where $c \neq 0$ is some constant.

By the root-locus result, for $\zeta$ large enough, all eigenvalues are distinct, and we have

$$
\begin{aligned}
\sqrt{p_{\text {ring }}^{(\zeta, N)}(i, j)} & =\sum_{k<N}\left|\left\langle i \mid v_{k}(\zeta)\right\rangle\left\langle v_{k}(\zeta) \mid j\right\rangle\right|+\left|\left\langle i \mid v_{N}(\zeta)\right\rangle\left\langle v_{N}(\zeta) \mid j\right\rangle\right| \\
& =\sqrt{p_{\text {chain }}^{(N-1)}(i, j)}+\left|\left\langle i \mid v_{N}(\zeta)\right\rangle\left\langle v_{N}(\zeta) \mid j\right\rangle\right|
\end{aligned}
$$

where the second equality is understood as the $\zeta \rightarrow \infty$ limit. To complete the proof, it therefore remains to look at $\left|v_{N}(\zeta)\right\rangle$.

The last $k=N$ eigenequation easily implies that $\zeta\left|v_{N}(\zeta)\right\rangle_{1: N-1}$ remains bounded as $\zeta \rightarrow \infty$. Therefore, $\lim _{\zeta \rightarrow \infty}\left|v_{N}(\zeta)\right\rangle_{1: N-1}=0$. To normalize the eigenvector, we take $\lim _{\zeta \rightarrow \infty}\left|v_{N}(\zeta)\right\rangle_{N}=1$. The latter together with (35) proves the theorem.

Thus, we have a systematic way to compute the asymptotic transfer probability of a ring with high bias from the transfer probability of a chain without bias.

Example 10 (Dynamic Routing.) As an illustration of how these results can be used, consider a ring of size $N=9$. The maximum transfer fidelities between nodes $i \neq j$ for this ring without bias are quite low, 0.4094 and 0.4444 . However, applying a large bias to node 9 changes the maximum transfer fidelities. In particular, the maximum transfer fidelity between nodes 1 and 8,2 and 7, 3 and 6 and 4 and 5 now approaches 1. Figure 6 shows a visual representation of the transfer fidelities for the ring without bias (left) and with bias (right). This result is consistent with Theorem 5, as using Lemma 2, it is easily verified that

$$
\sqrt{p_{\text {ring }}^{(8)}(i, 9-i)}=\frac{2}{9} \sum_{k=1}^{8}\left|\sin \left(\frac{\pi i k}{9}\right) \sin \left(\frac{\pi(9-i) k}{9}\right)\right|=1 \text {. }
$$

The example also shows that a finite bias is sufficient to enable almost perfect state transfer in practice, despite the fact that the ring only becomes a chain in the limit when an infinite bias is applied to node 9. We also used the LLL-inspired algorithm to estimate the transfer time as a function of the infidelity of the transfer. We note here that it was crucial to use the weighted LLL-algorithm to generate a range of simultaneous Diophantine approximations, which generally did not satisfy the parity constraints on the numerators, and to use the idea of combining approximations to satisfy the constraints. With this approach, we were able to find solutions satisfying all of the parity constraints on the numerators over a wide range of infidelities to 

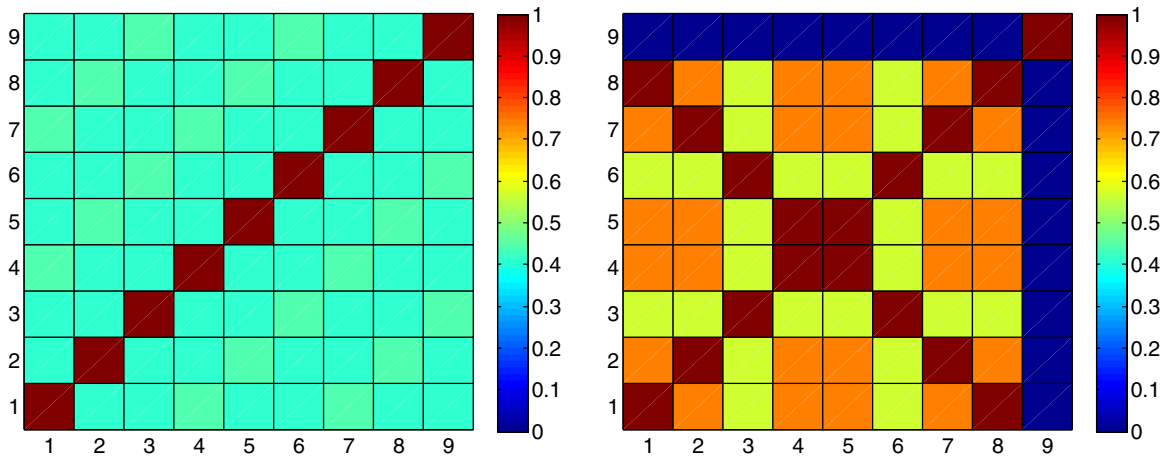

Fig. 6 Visual representation of maximum transfer fidelities for a ring of size 9 without bias (left) and the same ring with a finite bias applied to node 9 (right)
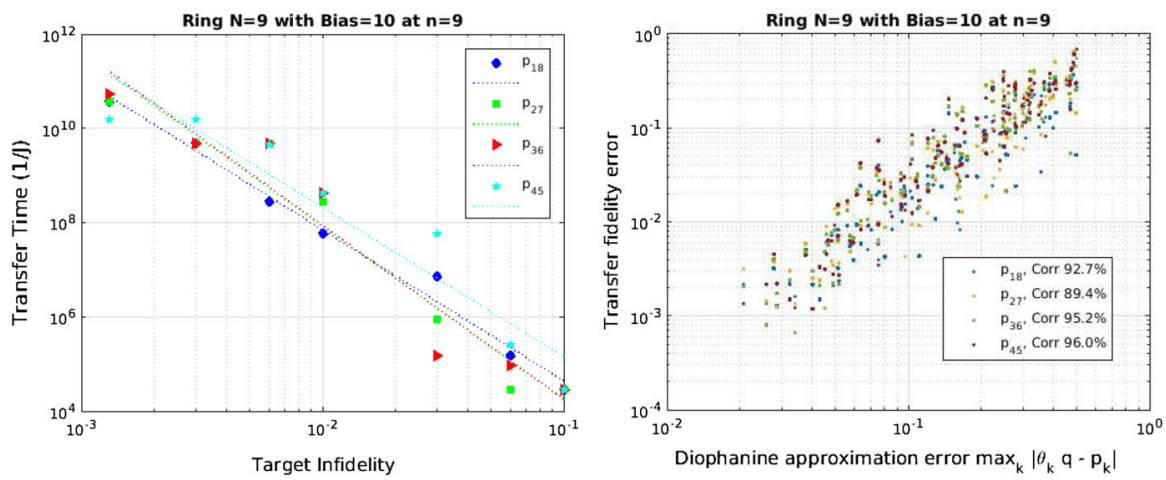

Fig. 7 Scaling of transfer times for transfers between nodes $(1,8),(2,7),(3,6)$ and $(4,5)$ for a ring of size $N=9$ with a bias of strength 10 (in units of $1 / J$ ) applied to node $n=9$ (left) and correlation of fidelity error (or infidelity) and Diophantine approximation error (right)

estimate the transfer times required as a function of the tolerated infidelity. The results, shown in Fig. 7 (left), suggest that high fidelities are indeed attainable for modest biases, and the apparent linearity of the data in the bilogarithmic plot still suggests a polynomial scaling. However, the actual transfer times are significantly higher in this case than in previous examples. We point out here that our algorithm is not guaranteed to find the shortest possible time although Fig. 7 (right) shows that there is a good correlation between the Diophantine approximation error and the observed infidelity of the transfer, as already anticipated by Eq. (20). Furthermore, the algorithm enables us to estimate necessary transfer times far beyond the regime accessible by brute-force numerical simulations.

This example shows how a dynamic routing scheme can be implemented to transfer information from any node in a ring to any other node with fidelity approaching unity by simply applying bias fields to different nodes. For transfer between nodes 1 and 8 , 2 and 7, 3 and 6, or 4 and 5, it suffices to apply a large bias to node 9 . If we wish to transfer information from node 1 to 4 , then translation invariance of the ring allows 
us to shift the labels by 2 , so that node 1 becomes 3 and 4 becomes 6 , and applying a bias to the new node 9 will enable the transfer.

Further reflection shows that we can achieve maximum transfer fidelities approaching unity for transfer between any pair of nodes in a ring of size $N$, provided $N$ is odd by simply biasing the node in the middle between the pair of spins. This is because in this case $N-2$ is odd, so there must be an odd number of spins along one path around the ring and an even number between the spins around the other. By applying the bias in the middle of the path with an odd number of spins, we asymptotically reach a chain with $N-1$ (even) spins. In this chain, the transfer probability between spins mirrored at the center is 1 , which is specifically true for the source and target spin with an even number of spins between them in the chain.

If $N$ is even instead, then the situation is more complicated. If there is an odd number of spins between source and target along the ring, then applying a bias at the middle creates an odd chain where source and target are connected with probability 1 as they are at mirror-symmetric positions in the ring. If there is an even number of spins between source and target, then applying a single bias cannot achieve perfect information transfer as the spins can never be at mirrored positions in the odd chain (which are the only ones in the chain perfectly connected). There are, however, multiple solutions to apply a bias at two spins that can asymptotically generate a suitable chain.

In practice, it may be possible and even preferable to simultaneously apply biases to several nodes instead of a single node to shape the overall potential landscape. This case is more difficult to treat analytically, but preliminary results [43] suggest that numerical optimization can be used in this case to optimize the applied biases to achieve significant reductions in the transfer times and the magnitude of the required bias fields, as well as to deal with practical issues such as leakage of the bias fields, i.e., the tendency of a bias applied to one node to also affect nearby nodes.

\section{Conclusion}

The concept of maximum transfer fidelity for information transfer between nodes in a network of interacting spins was introduced, and criteria for attainability of the bounds in terms of the transition frequencies of the network were given. Attainability was shown to be related, theoretically, to minimality of a linear flow and, computationally, to a translation on a torus. This last connection enabled us to derive upper bounds on the time required to realize transfer fidelities within $\epsilon_{\text {prob }}$ of the maximum transfer fidelity, for arbitrary $\epsilon_{\text {prob }}>0$, via the simultaneous Diophantine approximation. Algorithms were discussed to find the required approximations.

The ultimate aim of this analysis is to understand the intrinsic limitations of information transfer in spin networks and utilize this understanding to engineer networks with favorable bounds on the information transfer fidelities and dynamic attainability properties, so that high spin transfer fidelities can be attained in short times, enabling fast transfer and minimizing the effects of noise and decoherence. An advantage of our approach of combining general ITF bounds and asymptotic attainability conditions with an algorithm to estimate the time required to achieve transfer within a set margin of error, compared to engineering the spectrum of the network Hamiltonian to admit 
perfect state transfer, for example, is that the latter condition is generally a too strong requirement, as in practice there are always margins of error. Therefore, it makes more sense to ask how much time is required to achieve a certain transfer fidelity for a given acceptable margin of error $\epsilon$, and try to optimize the network topology, couplings or biases to achieve the best possible transfer times for the acceptable margins of error.

The general results were applied specifically to regular spin structures such as rings with uniform coupling. In this case, the information transfer infidelity prametric induced by maximum transfer fidelity takes on full significance as it can be shown to be a proper metric defining an information transfer infidelity geometry for the network, which is significantly different from the physical network geometry. The analysis shows that the intrinsic transfer fidelities for simple networks such as rings are often attainable asymptotically, but the times required to achieve high fidelities can be very long. The intrinsic bounds on the ITFs and transfer times can be favorably changed, however, by simple Hamiltonian engineering such as applying spatially distributed static bias fields. In particular, it was shown how such simple controls can be used to alter the information transfer fidelities and geometry of a network. It was demonstrated how this idea can be applied to enable or disable information transfer between a pair of nodes in the network. Simple bias controls are sufficient to direct information flow between nodes. By changing the biases, different transfers can be targeted, and thus a spin ring with fixed couplings can be turned into a simple quantum router for information encoded in excitations of a spin network.

Directions for future work include optimizing information transfer in spin networks via optimal control to achieve faster and more efficient dynamic routing in more complex spin networks. While this work focused on transfer of a single excitation, the concepts and analysis can also be applied to the case of encoding and simultaneous transfer of multiple excitations. This is interesting as it could increase the information transmission capacity of the network. Finally, although simulation results for similar spin systems suggest that some degree of intrinsic robustness of state transfer and the ability to mitigate the effects of noise, decoherence or fluctuations in the couplings via control $[10,11,44]$, the sensitivity of transfer fidelities with regard to noise and deleterious effect of the environment need to be investigated for specific physical realizations of spin networks.

Acknowledgments E. A. Jonckheere was partially supported by the Army Research Office (ARO) Multi University Research Initiative (MURI) grant W911NF-11-1-0268. S. G. Schirmer and F. C. Langbein acknowledge support from the Ser Cymru National Research Network on Advanced Engineering. SGS also acknowledges funding from a Royal Society Leverhulme Trust Senior Fellowship.

Open Access This article is distributed under the terms of the Creative Commons Attribution 4.0 International License (http://creativecommons.org/licenses/by/4.0/), which permits unrestricted use, distribution, and reproduction in any medium, provided you give appropriate credit to the original author(s) and the source, provide a link to the Creative Commons license, and indicate if changes were made.

\section{References}

1. Caruso, F., Giovannetti, V., Lupo, C., Mancini, S.: Quantum channels and memory effects. Rev. Mod. Phys. 86, 1203-1259 (2014) 
2. Awschalom, D.D., et al.: Quantum spintronics: engineering and manipulating atom-like spins in semiconductors. Science 339(6124), 1174-1179 (2013)

3. Bose, S.: Quantum communication through an unmodulated spin chain. Phys. Rev. Lett. 91, 07901 (2003)

4. Bose, S.: Quantum communication through spin chain dynamics: an introductory overview. Contemp. Phys. 48, 13-30 (2007)

5. Key, A.: A review of perfect, efficient, state transfer and its application as a constructive tool. Int. J. Quantum Inf. 08, 641 (2010)

6. Christandl, M., Datta, N., Ekert, A., Landahl, A.J.: Perfect state transfer in quantum spin networks. Phys. Rev. Lett. 92, 187902 (2004)

7. Christandl, M., Datta, N., Dorlas, T., Ekert, A., Kay, A., Landahl, A.: Perfect transfer of arbitrary states in quantum spin networks. Phys. Rev. A 71, 032312 (2005)

8. Greentree, A.D., Cole, J.H., Hamilton, A.R., Hollenberg, L.C.L.: Coherent electronic transfer in quantum dot systems using adiabatic passage. Phys. Rev. B 70, 235317 (2004)

9. Zueco, D., Galve, F., Kohler, S., Hänggi, P.: Quantum router based on ac control of qubit chains. Phys. Rev. A 80, 042303 (2009)

10. Schirmer, S.G., Pemberton-Ross, P.J.: Fast high-fidelity information transmission through spin-chain quantum wires. Phys. Rev. A 80, 030301 (2009)

11. Wang, X., Bayat, A., Bose, S., Schirmer, S.G.: Global control methods for Greenberger-HorneZeilinger-state generation on a one-dimensional ising chain. Phys. Rev. A 82, 012330 (2010)

12. Casaccino, A., Lloyd, S., Mancini, S., Severini, S.: Quantum state transfer through a qubit network with energy shifts and fluctuations. Int. J. Quantum Inf. 7(8), 1417-1427 (2009)

13. Jonckheere, E., Schirmer, S., Langbein, F.: Geometry and curvature of spin networks. In: IEEE MultiConference on Systems and Control, pp. 786-791, Denver (2011). doi:10.1109/CCA.2011.6044395. arXiv:1102.3208v1 [quant-ph]

14. Jonckheere, E., Schirmer, S., Langbein, F.: Quantum networks: the anti-core of spin chains. Quantum Inf. Process. 13, 1607-1637 (2014)

15. Wang, X., Pemberton-Ross, P., Schirmer, S.G.: Symmetry and controllability for spin networks with a single-node control. IEEE Trans. Autom. Control 57, 1945-1956 (2012)

16. Massey, A., Miller, S.J., Sinsheimer, J.: Distribution of eigenvalues of real symmetric palindromic Toeplitz matrices and circulant matrices. J. Theor. Probab. 20, 637-662 (2007)

17. Kaku, M.: Introduction to Superstrings, ser. Graduate Texts in Contemporary Physics. Springer, New York (1998)

18. Prakash, N.: Mathematical Perspectives on Theoretical Physics: A Journey from Black Holes to Superstrings. Tata McGraw-Hill, New Delhi (2000)

19. Katok, A., Hasselblatt, B.: Introduction to the Modern Theory of Dynamical Systems. Cambridge University Press, Cambridge (1997)

20. Lagarias, J.C.: The computational complexity of simultaneous Diophantine approximation problems. In: 23rd Annual Symposium on Foundations of Computer Science, 1982. SFCS '08, pp. 32-39 (1982)

21. Kovacs, A., Tihanyi, N.: Efficient computing of n-dimensional simultaneous Diophantine approximation problems. Acta Univ. Sapientiae Inform. 5(1), 16-34 (2013)

22. Simultaneous Diophantine approximation. http://www.math.tamu.edu/ Doug.Hensley/ SimultaneousDiophantine (2005)

23. Nowak, W.G.: On simultaneous Diophantine approximation. Rendiconti del Circolo Matematico di Palermo XXXIII, 456-460 (1984)

24. Chevallier, N.: A Survey of Best Simultaneous Diophantine Approximations. http://www.math.uha. fr/chevallier/publication/meilleures3 (2011)

25. Bosma, W., Smeets I.: Finding simultaneous Diophantine approximations with prescribed quality. In: Howe, E.W., Kedlaya, K.S., (eds.) ANTS X: Tenth Algorithmic Number Theory Symposium 2012, UC San Diego, July 9-13 2012. Preprint arXiv:1001.4455v1

26. Lagarias, J.C.: Best simultaneous Diophantine approximations II. Behavior of consecutive best approximations. Pac. J. Math. 102(1), 61-88 (1982)

27. Nowak, W.G.: A note on the simultaneous Diophantine approximation. Manuscr. Math. 36, 33-46 (1981)

28. Keng, H.L.: Introduction to Number Theory. Springer, Berlin (1982)

29. Koblitz, N.: A course in Number Theory and Cryptography, ser. Graduate Texts in Mathematics. Springer, Berlin (1988) 
30. Lenstra, K., Lenstra, H.W., Lovasz, L.: Factoring with rational coefficients. Math. Ann. 261(4), 515534 (1982)

31. Lagarias, J.C.: Geodesic multidimensional continued fractions. Proc. Lond. Math. Soc. 3(69), 464-488 (1994)

32. Arkhangel'skii, A.V., Pontryagin, L.S.: General topology i; basic concepts and constructions; dimension theory. In: Gramkrelidze, R.V. (ed.) Encyclopedia of Mathematical Sciences. Springer, Berlin (1990)

33. Aldrovandi, R., Pereira, J.G.: An Introduction to Geometrical Physics. World Scientific, Singapore (1995)

34. Ariaei, F., Lou, M., Jonckheere, E., Krishnamachari, B., Zuniga, M.: Curvature of indoor sensor network: clustering coefficient. EURASIP J. Wirel. Commun. Netw. 2008, 20 (2008), article ID 213185. doi: $10.1155 / 2008 / 2131185$

35. Wilson, W.A.: On semi-metric spaces. Am. J. Math. 53(2), 361-373 (1931). http://www.jstor.org/ stable/2370790

36. Shore, S.D.: Coherent distance functions. Topol. Proceed. 6, 405-422 (1981)

37. Blumenthal, L.M.: Some embedding theorems and characterization problems of distance geometry. Bull. Am. Math. Soc. 40, 321-338 (1943)

38. Jonckheere, E., Lou, M., Bonahon, F., Baryshnikov, Y.: Euclidean versus hyperbolic congestion in idealized versus experimental networks. Internet Math. 7(1), 1-27 (2011)

39. Wang, C., Jonckheere, E., Banirazi, R.: Wireless network capacity versus Ollivier-Ricci curvature under Heat Diffusion (HD) protocol. In: American Control Conference (ACC), pp. 3536-3541, Portland, June 04-06 2014. http://eudoxus2.usc.edu

40. Blumenthal, L.M.: Theory and Applications of Distance Geometry. Oxford at the Clarendon Press, London (1953)

41. Jonckheere, E., Langbein, F.C., Schirmer, S.G.: Curvature of quantum rings. In: Proceedings of the 5th International Symposium on Communications, Control and Signal Processing (ISCCSP 2012), Rome, May 2-4 2012. doi:10.1109/ISCCSP.2012.6217863

42. Gromov, M.: Hyperbolic groups. In: Gersten, S.M. (ed.) Essays in Group Theory, ser. Mathematical Sciences, pp. 75-263. Springer, New York (1987)

43. Langbein, F., Schirmer, S., Jonckheere, E.: Time-optimal information transfer in spintronic networks. to appear. In: Proceedings of IEEE CDC (2015), Preprint arXiv:1508.00928

44. Burgarth, D.: Quantum state transfer and time-dependent disorder in quantum chains. Eur. Phys. J. Special Top. 151(1), 147-155 (2007) 